\title{
The crystal structures of kidwellite and 'laubmannite', two complex fibrous iron phosphates
}

\section{U. Kolitsch*}

Institut für Mineralogie und Kristallographie, Universität Wien, Geozentrum, Althanstrasse 14, A-1090 Wien, Austria

\section{ABSTRACT}

The previously unknown, complex crystal structures of two fibrous ferric iron phosphate minerals have been solved using single-crystal X-ray diffraction data. The structure of a slightly arsenatian kidwellite has been refined in space group $P 2 / c\left(a=20.117(4), b=5.185(1), c=13.978(3) \AA, \beta=107.07(3)^{\circ}, V\right.$ $=1393.8(5) \AA^{3}, Z=2$ ) to $R 1=5.21 \%$; a revision of both space group symmetry and chemical formula is proposed. The idealized formula is $\mathrm{Na}\left(\mathrm{Fe}^{3+}, M\right)_{9+x}(\mathrm{OH})_{11}\left(\mathrm{H}_{2} \mathrm{O}\right)_{3}\left(\mathrm{PO}_{4}\right)_{6}$, where $M=\mathrm{Fe}^{3+}, \mathrm{Cu}^{2+}$ or other metal cation, and $x \approx 0.3$. The structure of a slightly arsenatian 'laubmannite' (as defined by Moore, 1970) has been refined in space group Pbcm $(a=5.172(1), b=13.999(3), c=31.083(6) \AA, V=$ $\left.2250.5(8) \AA^{3}, Z=4\right)$ to $R 1=3.14 \%$. The revised, idealized formula is $\left(\mathrm{Fe}^{3+}, \mathrm{Fe}^{2+}, M\right)_{8+x}\left(\mathrm{OH}, \mathrm{H}_{2} \mathrm{O}\right)_{9}(-$ $\left.\mathrm{H}_{2} \mathrm{O}\right)_{2}\left(\mathrm{PO}_{4}\right)_{5}$, where $M=\mathrm{Fe}^{3+}, \mathrm{Cu}^{2+}$ or other metal cation, and $x \approx 0.1$. The framework structures of both minerals are similar. Dominant building units are dimers composed of face- and edge-sharing $\mathrm{FeO}_{6}$ octahedra. Whereas kidwellite contains an additional trimer built of three corner-sharing $\mathrm{FeO}_{6}$ octahedra, 'laubmannite' instead contains a dimer built of two corner-sharing $\mathrm{FeO}_{6}$ octahedra. Kidwellite contains only trivalent iron, while one of the Fe sites in 'laubmannite' is occupied by a mixture of $\mathrm{Fe}^{3+}$ and $\mathrm{Fe}^{2+}$ in a 1:1 ratio. In both structures, the $\mathrm{FeO}_{6}$-based building units are linked via corners to $\mathrm{PO}_{4}$ tetrahedra; the $M$ sites are located in narrow channels and have very low occupancies $(\sim 2$ to $7 \%)$ and strongly distorted [6]- or [5+1]-coordinations. Close structural relations between kidwellite and 'laubmannite', and other fibrous iron phosphates explain observations of epitaxial intergrowths of them.

KeYwords: kidwellite, laubmannite, crystal structure, crystal chemistry, fibrous iron phosphates.

\section{Introduction}

KIDWELLITE and 'laubmannite' are two fibrous ferric iron phosphates which occur as late-stage or secondary minerals in a variety of environments, and are often associated with other iron phosphates such as dufrénite, rockbridgeite, beraunite, chalcosiderite, cacoxenite and strengite. The present work reports the previously unknown crystal structures of both minerals (based on single-crystal X-ray intensity data), and discusses their revised formulae, crystalchemical characteristics and the occurrence of epitaxial intergrowths.

*E-mail: uwe.kolitsch@univie.ac.at

DOI: $10.1180 / 0026461046810177$
A preliminary account of the results of a structure determination of a different 'laubmannite' crystal has already been given (Kolitsch, 2001; $R 1=6.55 \%$ ); the present results confirm and improve the earlier data. The present work is part of efforts to unravel the crystal chemistry of several complex and poorly characterized fibrous iron phosphates. In a previous study (Kolitsch, 1999), it was shown by X-ray powder diffraction and transmission electron microscopy that meurigite, $\mathrm{KFe}_{7}^{3+}\left(\mathrm{PO}_{4}\right)_{5}(\mathrm{OH})_{7} \cdot 8 \mathrm{H}_{2} \mathrm{O}$ (Birch et al., 1996) is identical to the earlier described phosphofibrite, $\mathrm{KCuFe}_{15}^{3+}\left(\mathrm{PO}_{4}\right)_{12}(\mathrm{OH})_{12} \cdot 12 \mathrm{H}_{2} \mathrm{O}$ (Walenta and Dunn, 1984). Unfortunately, no crystals of meurigite/phosphofibrite suitable for a single-crystal determination of their atomic arrangement have been found yet. 


\section{Previous work}

\section{Kidwellite}

Kidwellite was originally described by Moore and Ito (1978) from novaculite deposits in Arkansas, as well as from several other worldwide localities. A preliminary study of the species had used the designation 'Mineral A' (Moore, 1970). The mineral forms pale greenish, greenish yellow, greenish white or bright yellow radiating aggregates of small acicular to fibrous crystals. From single-crystal studies, Moore and Ito (1978) deduced space group $A 2 / m, A m$ or $A 2$, and the unit-cell parameters $a=20.61(7), b=5.15(1), c=$ 13.75(6) $\AA, \beta=112.64(15)^{\circ}, V=1347 \AA^{3}$. The acicular crystals are elongated along [010]. Chemical analyses gave the simplified formula $\mathrm{NaFe}_{9}^{3+}(\mathrm{OH})_{10}\left(\mathrm{PO}_{4}\right)_{6} \cdot 5 \mathrm{H}_{2} \mathrm{O}$ (Moore and Ito, 1978).

Moore (1970) suggested that the crystal structure of kidwellite contains " $h$-clusters", a designation he had coined for clusters composed of three face-sharing $\left(\mathrm{Fe}^{2+}, \mathrm{Fe}^{3+}\right) \mathrm{O}_{6}$ octahedra. The existence of ' $h$-clusters' was also inferred by Moore (1970) for the crystal structure of 'laubmannite' (see below).

Further studies of kidwellite are scarce, although occurrences of the mineral are numerous (e.g. Anthony et al., 2000). On studying an impurity-free sample from a French locality using X-ray powder diffraction, Walenta and Binder (1980) noted a reflection at $d=5.13 \AA$ $\left(I / I_{100}=0.3\right)$ which is not included in the powder data listed by Moore and Ito (1978), but is present in the earlier data given by Moore (1970), with $I / I_{100}=0.5$. Walenta and Binder (1980) pointed out that this reflection can be indexed as 010 , although this would contradict the $A$-centred monoclinic cell reported by Moore and Ito (1978). Infrared spectra for kidwellite from Cornwall were reported by Braithwaite and Corke (1980) who observed a sharp absorption at $3570 \mathrm{~cm}^{-1}$ and broad bands at 3300 and $3170 \mathrm{~cm}^{-1}(\mathrm{O}-\mathrm{H}$ stretching vibrations), as well as a broad band at $1635 \mathrm{~cm}^{-1}\left(\mathrm{H}_{2} \mathrm{O}\right.$ bending mode). The features of the $\mathrm{P}-\mathrm{O}$ vibrational bands indicated distorted $\mathrm{PO}_{4}$ groups (Braithwaite and Corke, 1980). A poorly resolved infrared (IR) spectrum of a kidwellite sample from Germany was given by Dietrich (1978b).

An electron microprobe analysis of kidwellite sprays from the Broken Hill deposit, NSW, Australia, yielded the formula $\left(\mathrm{Na}_{0.9} \mathrm{Ca}_{0.05}\right)_{\Sigma 0.95}\left(\mathrm{Fe}_{8.1}^{3+} \mathrm{Al}_{0.6} \mathrm{Zn}_{0.2} \mathrm{Cu}_{0.03}\right)_{\Sigma 8.93}$
$\left[\left(\mathrm{PO}_{4}\right)_{5.4}\left(\mathrm{AsO}_{4}\right)_{0.6}\right]_{\Sigma 6.00}(\mathrm{OH})_{9.4} \cdot 7.1 \mathrm{H}_{2} \mathrm{O}$ (Birch, 1990), based on the assumption that all $\mathrm{Fe}$ is trivalent and with $\mathrm{OH}$ calculated from chargebalance $\left(\mathrm{H}_{2} \mathrm{O}\right.$ content by difference). A sample from the Clara mine, Black Forest, Germany, was also found to contain considerable amounts of As, and additionally very minor $\mathrm{Cu}$ and $\mathrm{Cl}(?)$. Semiquantitative SEM-EDS analyses of a kidwellite from the Lausitz region, Germany, indicated that up to $\sim 25 \%$ of the Fe may be substituted by $\mathrm{Al}$ (Witzke and Giesler, 1997).

\section{Laubmannite}

The fibrous ferric iron phosphate laubmannite has a more complicated history than kidwellite. It was described as a new mineral species by Frondel (1949) from Buckeye Mountain, near Shady, Polk County, Arkansas, USA, where it occurs as thick, brownish to brownish green, colour-banded crusts with a parallel-fibrous structure upon 'limonite'. A wet-chemical analysis of laubmannite from the type locality by Hallowell in 1948, cited by Frondel (1949), gave (wt.\%) $\mathrm{CaO} 1.14, \mathrm{FeO} 2.07$, $\mathrm{MnO} 2.40, \mathrm{Fe}_{2} \mathrm{O}_{3}$ 57.88, $\mathrm{Al}_{2} \mathrm{O}_{3} 0.05, \mathrm{P}_{2} \mathrm{O}_{5}$ 25.95, $\mathrm{H}_{2} \mathrm{O}^{-} 0.44, \mathrm{H}_{2} \mathrm{O}^{+} 10.06$, sum 100.00. However, Frondel (1949) considered the $\mathrm{Fe}^{2+}: \mathrm{Fe}^{3+}$ ratio doubtful and assumed laubmannite to be isostructural with andrewsite on the basis of their similar X-ray powder diffraction patterns. Frondel (1949) proposed the formula $\left(\mathrm{Fe}^{2+}, \mathrm{Mn}^{2+}, \mathrm{Ca}\right)_{3} \mathrm{Fe}_{6}^{3+}$ $\left(\mathrm{PO}_{4}\right)_{4}(\mathrm{OH})_{12}$ for laubmannite. Andrewsite, however, was later discredited as a mixture of hentschelite and rockbridgeite, with minor chalcosiderite (Dunn, 1990).

A reinvestigation of 'laubmannite' was conducted by Moore (1970), using material from the type locality and from the Leveäniemi mine, Svappavaara mining district, Norbotten province, Sweden. By powder and single-crystal X-ray diffraction (XRD) film methods, he showed that his 'laubmannite' is orthorhombic, with $a=13.95$, $b=30.77, c=5.16 \AA, V=2214.9 \AA^{3}$, and probable space group Pbma (a non-standard setting of space group $\mathrm{Pbcm}$ ). Moore (1970) describes the Arkansas material as "radial discoidal aggregates of fibers, rich yellowishgreen in color." The X-ray powder pattern redetermined by Moore (1970) was very different from that of Frondel (1949). Therefore, Moore (1970) concluded that Frondel's X-ray sample probably consisted of a mixture of major dufrénite and minor amounts of other phosphates. However, Moore (1970) also noted that the original 
chemical analysis would conform to the suggested formula of laubmannite but not to that of dufrénite. He concluded that the previous studies were not made on the very same material and that the mineral investigated by him was a distinct, unnamed species. From crystal-chemical and topological considerations he inferred that 'laubmannite' was, together with dufrénite, rockbridgeite, kidwellite (designated 'Mineral A' at that time), a member of a group of iron phosphates all of which contain clusters composed of three face-sharing $\left(\mathrm{Fe}^{2+}, \mathrm{Fe}^{3+}\right) \mathrm{O}_{6}$ octahedra (' $h$-clusters'). Moore (1970) also mentioned the theoretical deduction of an unpublished preliminary model of the crystal structure.

Subsequently, a re-examination of the type specimen of laubmannite (Frondel, 1949) by Dunn (1990) demonstrated that this specimen consists of a variable mixture of dufrénite, kidwellite and beraunite. Consequently, Dunn (1990) formally discredited laubmannite, although he also concluded that the 'laubmannite' mineral characterized by Moore (1970) is a unique but unnamed species. The mineral described by Moore (1970) was later identified from only a small number of other occurrences. It has been reported from Indian Mountain, Cherokee Co., Alabama, USA (Barwood, 1974), the Rotläufchen mine, Waldgirmes near Wetzlar, Germany (Dietrich, 1978a,b, 1982), the Clara mine, Central Black Forest, Germany (Walenta, 1981) and Auerbach, Oberpfalz, Germany (Geipel, 1982; see also Stanjek, 1983). The occurrence at the Leveäniemi Mine, Svappavaara, Sweden mentioned by Moore (1970) was later confirmed (Bjällerud, 1989). An occurrence in New Mexico was mentioned by Gibbs (1991), but the sample was subsequently found to be beraunite (Northrop, 1996). A poorly resolved infrared spectrum of a 'laubmannite' from Germany was given by Dietrich (1978b). No other new data were reported except for some semiquantitative chemical compositions (Kolitsch, 2001; see Experimental section).

\section{Oriented intergrowths}

Epitaxial intergrowths of kidwellite and 'laubmannite' with either each other or with other iron phosphates were observed by several authors. For instance, Moore (1970) describes 'laubmannite' intergrown with dufrénite at the crystal tips of the latter. Dietrich (1978b) observed that kidwellite needles from the Rotläufchen mine were grown on the (011) faces of hydrothermally altered rockbridgeite, with the $\sim 5.1 \AA$ axes of both minerals being parallel. The same observation was also made for tiny 'laubmannite' needles grown epitaxially on rockbridgeite, and for oriented overgrowths of dufrénite on rockbridgeite (Dietrich, 1978a,b). From Namibia, fibrous kidwellite in oriented overgrowths on older rockbridgeite was described by Keller (1985), albeit without giving details. Walenta (1981, 1992) observed that 'laubmannite' from the Clara mine is partly replaced by beraunite in such a manner that the $\sim 5.1 \AA$ axis ( $c$ axis of Moore, 1970) of 'laubmannite' is parallel to the $b$ axis $(\sim 5.1 \AA)$ of beraunite. Equivalent oriented intergrowths of kidwellite on laubmannite have been observed (Walenta, 1990, 1992). Dufrénite crystals which, at their tips, grade into kidwellite were described by Walenta (1995). Fibrous kidwellite grading into 'laubmannite' was observed by Walenta and Theye (2001).

\section{Experimental}

\section{Kidwellite}

The kidwellite sample used for the present study is from the Clara baryte and fluorite mine, Black Forest, Germany. Tufts of pale olive-greenish acicular crystals up to $\sim 0.5 \mathrm{~mm}$ long and $\sim 0.01 \mathrm{~mm}$ in diameter cover 'limonite' on a matrix of quartz and minor baryte. Semiquantitative chemical analyses of the kidwellite by conventional energy-dispersive X-ray spectrometry in a scanning electron microscope (SEM-EDS) on several carbon-coated crystals showed the presence of major Fe and P, and very minor $\mathrm{Al}, \mathrm{Cu}$ and $\mathrm{As}$. The analytical results are similar to those obtained by Walenta (1990) on a kidwellite sample from the same locality.

Preliminary investigations of several acicular crystals were done with a Nonius KappaCCD single-crystal diffractometer which was equipped with a $300 \mu \mathrm{m}$ diameter capillary-optics collimator to provide increased resolution. All crystals showed a primitive monoclinic unit cell, and the most suitable crystal was selected for an intensity data collection (for details see Table 1). The data were processed with the Nonius program suite DENZO-SMN and corrected for Lorentz, polarization, background and absorption effects. Observed extinctions and intensity statistics strongly suggested space group $P 2 / c$. The crystal structure was solved in space group $P 2 / c$ by direct 


\section{U. KOLITSCH}

TABLE 1. Crystal data, data-collection information and refinement details for kidwellite and 'laubmannite' (Moore, 1970) from the Clara mine, Black Forest, Germany.

\begin{tabular}{|c|c|c|}
\hline & Kidwellite & Laubmannite (sample LAUB2) \\
\hline \multicolumn{3}{|l|}{ Crystal data } \\
\hline Formula (idealized) & $\mathrm{Na}\left(\mathrm{Fe}^{3+}, M\right)_{9+x}(\mathrm{OH})_{11}\left(\mathrm{H}_{2} \mathrm{O}\right)_{3}\left(\mathrm{PO}_{4}\right)_{6}$ & $\left(\mathrm{Fe}^{3+}, \mathrm{Fe}^{2+}, M\right)_{8+x}\left(\mathrm{OH}, \mathrm{H}_{2} \mathrm{O}\right)_{9}\left(\mathrm{H}_{2} \mathrm{O}\right)_{2}\left(\mathrm{PO}_{4}\right)_{5}$ \\
\hline Space group & $P 2 / c$ (no. 13 ) & $P b c m($ no. 57$)$ \\
\hline$a, b, c(\AA)$ & $20.117(4), 5.185(1), 13.978(3)$ & 5.172(1), 13.999(3), 31.083(6) \\
\hline$\beta\left({ }^{\circ}\right)$ & $107.07(3)$ & - \\
\hline$V\left(\AA^{3}\right), Z$ & 1393.8(5), 2 & $2250.5(8), 4$ \\
\hline$\mu\left(\mathrm{mm}^{-1}\right), \rho_{\text {calc }}\left(\mathrm{g} \mathrm{cm}^{-3}\right)$ & $5.86,3.289$ & $6.00,3.372$ \\
\hline Absorption correction & $\begin{array}{l}\text { multi-scan (Otwinowsky } \\
\text { and Minor, 1997) }\end{array}$ & multi-scan (Otwinowsky and Minor, 1997) \\
\hline Crystal dimensions (mm) & $0.01 \times 0.01 \times 0.15$ & $0.05 \times 0.07 \times 0.18$ \\
\hline \multicolumn{3}{|c|}{ Data collection and refinement } \\
\hline Diffractometer & Nonius Kappa CCD system & Nonius Kappa CCD system \\
\hline$T(\mathrm{~K}) ; \lambda(\mathrm{Mo}-K \alpha)(\AA)$ & $293 ; 0.71073$ & $293 ; 0.71073$ \\
\hline Detector distance $(\mathrm{mm})$ & 34 & 44 \\
\hline Rotation axis; width $\left({ }^{\circ}\right)$ & $\varphi, \omega ; 1.0$ & $\varphi, \omega ; 0.8$ \\
\hline Total no. of frames & 544 & 716 \\
\hline Collect. time/degree (s) & 460 & 300 \\
\hline $2 \theta_{\max }\left({ }^{\circ}\right)$ & 52.78 & 55.00 \\
\hline$h, k, l$ ranges & $-25 \rightarrow 24,-6 \rightarrow 6,-17 \rightarrow 17$ & $-6 \rightarrow 6,-17 \rightarrow 18,-39 \rightarrow 40$ \\
\hline Total refl.s measured & 5265 & 4773 \\
\hline Unique reflections & $2850\left(R_{\text {int }}=4.55 \%\right)$ & $2627\left(R_{\text {int }}=1.46 \%\right)$ \\
\hline$R 1(F), \mathrm{w} R 2\left(F^{2}\right)^{*}$ & $5.21 \%, 13.92 \%$ & $3.14 \%, 9.09 \%$ \\
\hline 'Observed' reflections & $1982\left[F_{\mathrm{o}}>4 \sigma\left(F_{\mathrm{o}}\right)\right]$ & $2239\left[F_{\mathrm{o}}>4 \sigma\left(F_{\mathrm{o}}\right)\right]$ \\
\hline No. of refined parameters & 264 & 233 \\
\hline Extinct. coefficient & $0.0004(4)$ & - \\
\hline $\mathrm{GoF}$ & 1.079 & 1.136 \\
\hline$(\Delta / \sigma)_{\max }$ & 0.0001 & 0.001 \\
\hline$\Delta \rho_{\min }, \Delta \rho_{\max }\left(\mathrm{e} / \AA^{3}\right)$ & $-1.01,1.12$ & $-0.84,0.83$ \\
\hline
\end{tabular}

Unit-cell parameters of kidwellite and 'laubmannite' were refined from 3090 and 3000 reflections, respectively. Scattering factors for neutral atoms were employed in the refinement.

${ }^{*} \mathrm{w}=1 /\left[\sigma^{2}\left(F_{o}{ }^{2}\right)+(0.07 \mathrm{P})^{2}+5.4 \mathrm{P}\right] ; P=\left(\left[\max\right.\right.$ of $\left(0\right.$ or $\left.\left.\left.F_{o}^{2}\right)\right]+2 F_{c}{ }^{2}\right) / 3$ (kidwellite) $\mathrm{w}=1 /\left[\sigma^{2}\left(F_{o}{ }^{2}\right)+(0.048 \mathrm{P})^{2}+7.2 \mathrm{P}\right] ; P=\left(\left[\max\right.\right.$ of $\left(0\right.$ or $\left.\left.\left.F_{o}{ }^{2}\right)\right]+2 F_{c}^{2}\right) / 3$ ('laubmannite')

methods (SHELXS-97, Sheldrick, 1997a), and the positions of all $\mathrm{Na}, \mathrm{Fe}, \mathrm{P}$ and $\mathrm{O}$ atoms were quickly located using subsequent differenceFourier maps. A full-matrix, anisotropic leastsquares refinement on $F^{2}$ (SHELXL-97, Sheldrick 1997b; see Table 1) showed that all three detected $\mathrm{P}$ sites were partly occupied by As, but to a different degree. From the Fourier difference maps, it was also apparent that there were three additional cation sites with very low occupancies and strongly distorted 'octahedral' coordinations. These sites were modelled as $\mathrm{Cu}$ atoms, and a final refinement step converged at $R 1=5.21 \%$ (Table 1). No $\mathrm{H}$ atoms could be located. The maximum peaks in the final difference-Fourier map were 1.12 and $-1.01 \mathrm{e} / \AA^{3}$. The refined positional and displacement parameters are given in Tables 2 and 3, and selected bond lengths and suggested hydrogen bonds in Table 4. A bond-valence analysis is given in Table 5. A list of observed and calculated structure factors (Table 6) has been deposited with the Principal Editor of Mineralogical Magazine and is available upon request.

\section{'Laubmannite'}

Two 'laubmannite' samples (as defined by Moore, 1970) were used for the present study, LAUB1 and LAUB2, both being from the Clara baryte and 
TABLE 2. Fractional atomic coordinates and isotropic displacement parameters (in $\AA^{2}$ ) for kidwellite.

\begin{tabular}{|c|c|c|c|c|c|}
\hline Atom & $x$ & $y$ & $z$ & $U_{\text {eq }} / U_{\text {iso }}$ & Occupancy \\
\hline $\mathrm{Fe}(1)$ & $-0.31930(5)$ & $-0.2542(2)$ & $0.19789(8)$ & $0.0080(3)$ & \\
\hline $\mathrm{Fe}(2)$ & $-0.17514(5)$ & $-0.2401(2)$ & $0.18336(8)$ & $0.0088(3)$ & \\
\hline $\mathrm{Fe}(3)$ & $-0.43127(6)$ & $-0.0041(2)$ & $0.96833(8)$ & $0.0151(3)$ & \\
\hline $\mathrm{Fe}(4)$ & $-0.19236(6)$ & $-0.0145(2)$ & $0.92828(8)$ & $0.0131(3)$ & \\
\hline $\mathrm{Fe}(5)$ & 0.0 & 0.0 & 0.0 & $0.0145(4)$ & \\
\hline $\mathrm{P}(1)$ & $-0.27367(8)$ & $0.2432(3)$ & $0.07103(12)$ & $0.0088(4)$ & $\mathrm{P}_{0.857(3)} \mathrm{As}_{0.143(3)}$ \\
\hline $\mathrm{P}(2)$ & $-0.10006(9)$ & $-0.7385(3)$ & $0.29965(13)$ & $0.0095(5)$ & $\mathrm{P}_{0.936(3)} \mathrm{As}_{0.064(3)}$ \\
\hline $\mathrm{P}(3)$ & $-0.35088(10)$ & $-0.2392(4)$ & $0.81893(13)$ & $0.0090(5)$ & $\mathrm{P}_{0.969(3)} \mathrm{As}_{0.031(3)}$ \\
\hline $\mathrm{Na}$ & 0.0 & $0.1807(14)$ & 0.75 & $0.0496(17)$ & \\
\hline$M(1)$ & 0.0 & 0.5 & 0.0 & $0.018(7)$ & $0.074(6)^{*}$ \\
\hline$M(2)$ & $-0.4316(8)$ & $0.509(3)$ & $0.9672(11)$ & $0.020 * *$ & $0.067(2)^{*}$ \\
\hline$M(3)$ & $-0.1943(7)$ & $0.515(3)$ & $0.9266(10)$ & $0.016(5)$ & $0.074(4) *$ \\
\hline $\mathrm{O}(1)$ & $-0.3506(3)$ & $0.2407(10)$ & $0.0091(4)$ & $0.0150(12)$ & \\
\hline $\mathrm{O}(2)$ & $-0.2218(3)$ & $0.2528(9)$ & $0.0089(4)$ & $0.0116(11)$ & \\
\hline $\mathrm{O}(3)$ & $-0.2589(3)$ & $-0.0084(10)$ & $0.1372(4)$ & $0.0114(11)$ & \\
\hline $\mathrm{O}(4)$ & $-0.2575(3)$ & $-0.5138(10)$ & $0.1425(4)$ & $0.0107(11)$ & \\
\hline $\mathrm{O}(5)$ & $-0.1000(3)$ & $-0.4805(11)$ & $0.2456(4)$ & $0.0178(13)$ & \\
\hline $\mathrm{O}(6)$ & $0.0290(3)$ & $0.2277(11)$ & $0.1213(4)$ & $0.0228(14)$ & \\
\hline $\mathrm{O}(7)$ & $-0.1591(3)$ & $-0.2668(10)$ & $0.8489(4)$ & $0.0129(11)$ & \\
\hline $\mathrm{O}(8)$ & $-0.1104(3)$ & $0.0381(11)$ & $0.2227(4)$ & $0.0223(14)$ & \\
\hline $\mathrm{O}(9)$ & $-0.2813(3)$ & $-0.1897(11)$ & $0.8954(4)$ & $0.0226(14)$ & \\
\hline $\mathrm{O}(10)$ & $-0.3550(3)$ & $-0.4954(10)$ & $0.2708(4)$ & $0.0204(13)$ & \\
\hline $\mathrm{O}(11)$ & $-0.4098(3)$ & $-0.2232(10)$ & $0.8678(4)$ & $0.0142(12)$ & \\
\hline $\mathrm{O}(12)$ & $-0.3642(3)$ & $0.0368(10)$ & $0.2340(4)$ & $0.0179(13)$ & \\
\hline $\mathrm{Oh}(13)$ & $-0.3916(3)$ & $-0.2733(10)$ & $0.0697(4)$ & $0.0111(11)$ & \\
\hline $\mathrm{Oh}(14)$ & $-0.1624(3)$ & $-0.2658(10)$ & $0.0485(4)$ & $0.0112(11)$ & \\
\hline $\mathrm{Oh}(15)$ & $-0.2189(3)$ & $-0.2410(9)$ & $0.3053(4)$ & $0.0107(11)$ & \\
\hline $\mathrm{Oh}(16)$ & $-0.4729(3)$ & $0.1709(12)$ & $0.0667(4)$ & $0.0218(14)$ & \\
\hline $\mathrm{Ow}(17)$ & $-0.4767(3)$ & $0.2842(11)$ & $0.8614(4)$ & $0.0250(14)$ & \\
\hline $\mathrm{Oh}(18)$ & $-0.0955(3)$ & $0.1400(12)$ & $0.9762(4)$ & $0.0210(13)$ & \\
\hline Ow(19) & $0.0278(3)$ & $0.2856(11)$ & $0.9185(4)$ & $0.0236(14)$ & $\underset{* * *}{\left(\mathrm{OH}_{0.5} \mathrm{H}_{2} \mathrm{O}_{0.5}\right)}$ \\
\hline
\end{tabular}

Oxygens of hydroxyl groups are designated as Oh, those of water molecules as Ow.

* Occupancy factors refined based on assumption that $M$ sites are occupied by $\mathrm{Cu}$ (see text).

$* *$ fixed. $* * *$ see text

fluorite mine, Black Forest, Germany. Several 'laubmannite' samples from other reported localities (Leveäniemi mine, Svappavaara mining district, Sweden; several occurrences in Polk Co., Arkansas, USA) were also investigated, but only very thin fibrous crystals were found, unsuitable for single-crystal data collections. In some cases the samples also turned out to be other misidentified phosphates (e.g. fibrous dufrénite or rockbridgeite). A crystal from sample LAUB1 was used for the original solution of the structure, a preliminary report of which was given in Kolitsch (2001); a crystal from LAUB2, which had a better crystal quality, was used for an improved refinement of the structure. Sample descriptions and data collections will be described for both samples, although only the (improved) results from LAUB2 will be listed in the Tables.

On the thumbnail-sized sample LAUB1, 'laubmannite' forms dark greenish to greenish brown, flat-lying radiating sprays of indistinct prismatic crystals up to $\sim 0.5 \mathrm{~mm}$ in length. The sprays are associated with tiny, pale greenish chalcosiderite crystals and with very pale greenish, fibrous sprays of another Fe-phosphate (probably kidwellite). Sample LAUB2 consisted of several small broken hemispheres (diameter $\sim 0.5 \mathrm{~mm}$ ) with a radiating internal structure. The 


\section{U. KOLITSCH}

TABle 3. Anisotropic displacement parameters for kidwellite.

\begin{tabular}{|c|c|c|c|c|c|c|}
\hline Atom & $U_{11}$ & $U_{22}$ & $U_{33}$ & $U_{23}$ & $U_{13}$ & $U_{12}$ \\
\hline $\mathrm{Fe}(1)$ & $0.0079(5)$ & $0.0075(6)$ & $0.0094(5)$ & $0.0000(5)$ & $0.0037(4)$ & $-0.0004(5)$ \\
\hline $\mathrm{Fe}(2)$ & $0.0087(5)$ & $0.0094(6)$ & $0.0091(5)$ & $0.0012(5)$ & $0.0039(4)$ & $0.0010(5)$ \\
\hline $\mathrm{Fe}(3)$ & $0.0151(6)$ & $0.0154(6)$ & $0.0150(6)$ & $-0.0006(5)$ & $0.0050(5)$ & $0.0016(5)$ \\
\hline $\mathrm{Fe}(4)$ & $0.0125(6)$ & $0.0140(6)$ & $0.0136(6)$ & $-0.0008(5)$ & $0.0048(5)$ & $0.0007(5)$ \\
\hline $\mathrm{Fe}(5)$ & $0.0124(8)$ & $0.0155(9)$ & $0.0153(8)$ & $-0.0018(7)$ & $0.0035(7)$ & $-0.0010(7)$ \\
\hline $\mathrm{P}(1)$ & $0.0091(8)$ & $0.0087(8)$ & $0.0096(8)$ & $-0.0001(7)$ & $0.0043(6)$ & $-0.0002(7)$ \\
\hline $\mathrm{P}(2)$ & $0.0096(9)$ & $0.0094(9)$ & $0.0097(9)$ & $0.0010(7)$ & $0.0033(7)$ & $0.0005(8)$ \\
\hline $\mathrm{P}(3)$ & $0.0081(9)$ & $0.0105(10)$ & $0.0094(9)$ & $-0.0012(8)$ & $0.0043(7)$ & $-0.0016(8)$ \\
\hline $\mathrm{Na}$ & $0.033(3)$ & $0.088(5)$ & $0.029(3)$ & 0.0 & $0.012(3)$ & 0.0 \\
\hline $\mathrm{O}(1)$ & $0.014(3)$ & $0.014(3)$ & $0.016(3)$ & $0.001(2)$ & $0.005(2)$ & $-0.001(3)$ \\
\hline $\mathrm{O}(2)$ & $0.011(3)$ & $0.011(3)$ & $0.014(3)$ & $0.000(2)$ & $0.005(2)$ & $0.003(2)$ \\
\hline $\mathrm{O}(3)$ & $0.010(3)$ & $0.010(3)$ & $0.015(3)$ & $0.007(2)$ & $0.006(2)$ & $0.004(2)$ \\
\hline $\mathrm{O}(4)$ & $0.007(3)$ & $0.011(3)$ & $0.015(3)$ & $0.000(2)$ & $0.004(2)$ & $0.003(2)$ \\
\hline $\mathrm{O}(5)$ & $0.011(3)$ & $0.022(3)$ & $0.022(3)$ & $0.011(3)$ & $0.008(2)$ & $0.004(3)$ \\
\hline $\mathrm{O}(6)$ & $0.014(3)$ & $0.033(4)$ & $0.020(3)$ & $-0.012(3)$ & $0.002(3)$ & $-0.001(3)$ \\
\hline $\mathrm{O}(7)$ & $0.011(3)$ & $0.014(3)$ & $0.014(3)$ & $-0.005(2)$ & $0.005(2)$ & $0.001(2)$ \\
\hline $\mathrm{O}(8)$ & $0.020(3)$ & $0.023(3)$ & $0.029(3)$ & $-0.011(3)$ & $0.014(3)$ & $-0.007(3)$ \\
\hline $\mathrm{O}(9)$ & $0.011(3)$ & $0.029(4)$ & $0.025(3)$ & $-0.002(3)$ & $0.000(3)$ & $-0.008(3)$ \\
\hline $\mathrm{O}(10)$ & $0.019(3)$ & $0.016(3)$ & $0.030(3)$ & $0.008(3)$ & $0.014(3)$ & $0.002(3)$ \\
\hline $\mathrm{O}(11)$ & $0.013(3)$ & $0.021(3)$ & $0.011(3)$ & $-0.004(2)$ & $0.007(2)$ & $0.002(2)$ \\
\hline $\mathrm{O}(12)$ & $0.018(3)$ & $0.014(3)$ & $0.021(3)$ & $-0.009(2)$ & $0.007(3)$ & $0.002(2)$ \\
\hline $\mathrm{Oh}(13)$ & $0.010(3)$ & $0.014(3)$ & $0.006(3)$ & $0.001(2)$ & $-0.002(2)$ & $-0.001(2)$ \\
\hline $\mathrm{Oh}(14)$ & $0.011(3)$ & $0.012(3)$ & $0.010(3)$ & $0.002(2)$ & $0.003(2)$ & $0.000(2)$ \\
\hline $\mathrm{Oh}(15)$ & $0.016(3)$ & $0.010(3)$ & $0.006(2)$ & $0.000(2)$ & $0.003(2)$ & $0.001(2)$ \\
\hline $\mathrm{Oh}(16)$ & $0.017(3)$ & $0.028(4)$ & $0.026(3)$ & $0.002(3)$ & $0.014(3)$ & $-0.001(3)$ \\
\hline Ow(17) & $0.024(3)$ & $0.026(4)$ & $0.022(3)$ & $0.010(3)$ & $0.001(3)$ & $0.000(3)$ \\
\hline $\mathrm{Oh}(18)$ & $0.014(3)$ & $0.017(3)$ & $0.030(4)$ & $0.001(3)$ & $0.004(3)$ & $0.003(3)$ \\
\hline Ow(19) & $0.024(3)$ & $0.031(4)$ & $0.021(3)$ & $0.009(3)$ & $0.015(3)$ & $0.006(3)$ \\
\hline
\end{tabular}

central, dark greenish zone of these hemispheres graded into a narrow, greenish to yellow-brown, altered outer zone, followed by a very narrow, brown, completely oxidized zone.

Semiquantitative chemical analyses of both 'laubmannite' samples and the associated phosphates were done by SEM-EDS on carbon-coated grains. LAUB1 contains major $\mathrm{Fe}, \mathrm{P}$ and trace amounts of $\mathrm{Cu}$ and $\mathrm{Mn}$. The associated kidwellite(?) contains trace amounts of $\mathrm{Cu}$, whereas the spectrum of chalcosiderite did not show any impurity elements. The dark greenish part of LAUB2 contains major $\mathrm{Fe}, \mathrm{P}$ and very small amounts of $\mathrm{As}, \mathrm{Cu}$ and $\mathrm{Al}$. The altered outer zone has practically the same composition, whereas the outermost oxidized zone contains only $\mathrm{Fe}$ and traces of P. Optical properties of LAUB1 were investigated in polarized light. Cleavage fragments showed a lath-like shape, straight extinction and negative elongation. Strong, bluish green to greenish brown pleochroic colours are observed, which can be attributed to $\mathrm{Fe}^{2+}-\mathrm{Fe}^{3+}$ charge transfer colouration (Moore, 1970). Optical properties of LAUB2 were not studied.

Preliminary investigations of several crystal fragments from LAUB1 with a Nonius KappaCCD single-crystal diffractometer revealed a primitive orthorhombic unit-cell with parameters very close to those determined by Moore (1970). All fragments were elongated parallel to the short $5.16 \AA$ axis, in agreement with Moore (1970). Most fragments showed, however, imperfect quality and were unsuitable for data collection. Eventually, a tiny elongate tabular cleavage fragment with suitable, albeit imperfect quality could be found. LAUB2 immediately provided a dark greenish cleavage fragment of good crystal quality. Orientation studies showed that the cleavage is perpendicular to the $\sim 14 \AA$ axis.

Both fragments were mounted on a Nonius KappaCCD diffractometer equipped with a $300 \mu \mathrm{m}$ diameter capillary-optics collimator to 


\section{CRYSTAL STRUCTURES OF KIDWELLITE AND 'LAUBMANNITE'}

TABLE 4. Selected bond distances $(\AA)$ for the coordination polyhedra in kidwellite.

\begin{tabular}{|c|c|c|c|}
\hline $\mathrm{Fe}(1)-\mathrm{O}(10)$ & $1.883(5)$ & $\mathrm{Fe}(2)-\mathrm{O}(8)$ & $1.914(6)$ \\
\hline$-\mathrm{O}(12)$ & $1.901(5)$ & $-O(5)$ & $1.955(5)$ \\
\hline$-\mathrm{Oh}(13)$ & $1.950(5)$ & $-\mathrm{Oh}(14)$ & $1.980(5)$ \\
\hline$-\mathrm{O}(3)$ & $2.105(5)$ & $-\mathrm{O}(3)$ & $2.015(5)$ \\
\hline$-\mathrm{O}(4)$ & $2.127(5)$ & $-\mathrm{O}(4)$ & $2.128(5)$ \\
\hline$-\mathrm{Oh}(15)$ & $2.134(5)$ & $-\mathrm{Oh}(15)$ & $2.137(5)$ \\
\hline$<\mathrm{Fe}(1)-\mathrm{O}>$ & 2.017 & $<\mathrm{Fe}(2)-\mathrm{O}>$ & 2.022 \\
\hline $\mathrm{Fe}(3)-\mathrm{O}(11)$ & $1.951(5)$ & $\mathrm{Fe}(4)-\mathrm{O}(9)$ & $1.937(6)$ \\
\hline$-\mathrm{Oh}(13)$ & $1.983(5)$ & $-O(7)$ & $1.956(5)$ \\
\hline$-\mathrm{O}(1)$ & $2.007(5)$ & $-\mathrm{O}(2)$ & $1.983(5)$ \\
\hline$-\mathrm{Oh}(16)$ & $2.023(6)$ & $-\mathrm{Oh}(18)$ & $2.029(6)$ \\
\hline$-\mathrm{Oh}(16)$ & $2.037(6)$ & $-\mathrm{Oh}(14)$ & $2.071(5)$ \\
\hline$-\mathrm{Ow}(17)$ & $2.122(6)$ & $-\mathrm{Oh}(15)$ & $2.111(5)$ \\
\hline$<\mathrm{Fe}(3)-\mathrm{O}>$ & 2.021 & $<\mathrm{Fe}(4)-\mathrm{O}>$ & 2.015 \\
\hline $\mathrm{Fe}(5)-\mathrm{Oh}(18) \times 2$ & $1.989(6)$ & $\mathrm{Na}-\mathrm{Ow}(19) \times 2$ & $2.320(6)$ \\
\hline$-\mathrm{O}(6) \times 2$ & $2.008(5)$ & $-\mathrm{O}(8) \times 2$ & $2.422(7)$ \\
\hline$-O w(19) \times 2$ & $2.044(6)$ & $-\mathrm{O}(5) \times 2$ & $2.529(7)$ \\
\hline$<\mathrm{Fe}(5)-\mathrm{O}>$ & 2.014 & $<\mathrm{Na}-\mathrm{O}>$ & 2.424 \\
\hline $\mathrm{P}(1)-\mathrm{O}(1)$ & $1.535(6)$ & $\mathrm{P}(2)-\mathrm{O}(5)$ & $1.536(6)$ \\
\hline$-\mathrm{O}(2)$ & $1.542(5)$ & $-\mathrm{O}(6)$ & $1.538(6)$ \\
\hline$-\mathrm{O}(3)$ & $1.576(5)$ & $-O(7)$ & $1.539(5)$ \\
\hline$-\mathrm{O}(4)$ & $1.581(5)$ & $-\mathrm{O}(8)$ & $1.553(6)$ \\
\hline$<\mathrm{P}(1)-\mathrm{O}>$ & 1.559 & $<\mathrm{P}(2)-\mathrm{O}>$ & 1.542 \\
\hline $\mathrm{P}(3)-\mathrm{O}(9)$ & $1.513(6)$ & $M(1)-\mathrm{Ow}(19) \times 2$ & $1.795(6)$ \\
\hline$-\mathrm{O}(10)$ & $1.524(6)$ & $-\mathrm{O}(6) \times 2$ & $2.152(6)$ \\
\hline$-\mathrm{O}(11)$ & $1.534(5)$ & $-\mathrm{Oh}(18) \times 2$ & $2.629(6)$ \\
\hline$-\mathrm{O}(12)$ & $1.548(5)$ & $<M(1)-\mathrm{O}>$ & 2.192 \\
\hline$<\mathrm{P}(3)-\mathrm{O}>$ & 1.530 & & \\
\hline$M(2)-\mathrm{Oh}(13)$ & $1.817(16)$ & $M(3)-\mathrm{O}(7)$ & $1.848(14)$ \\
\hline$-O w(17)$ & $1.892(16)$ & $-\mathrm{O}(2)$ & $1.960(14)$ \\
\hline$-\mathrm{O}(1)$ & 2.091(16) & $-\mathrm{Oh}(14)$ & $1.992(14)$ \\
\hline$-\mathrm{O}(11)$ & $2.101(16)$ & $-\mathrm{Oh}(15)$ & $2.154(14)$ \\
\hline$-\mathrm{Oh}(16)$ & $2.478(16)$ & $-\mathrm{O}(9)$ & $2.270(15)$ \\
\hline$-\mathrm{Oh}(16)$ & $2.524(16)$ & $-\mathrm{Oh}(18)$ & $2.719(14)$ \\
\hline$<M(2)-\mathrm{O}>$ & 2.151 & $<M(3)-\mathrm{O}>$ & 2.157 \\
\hline \multicolumn{4}{|c|}{ Tentative hydrogen bonds } \\
\hline $\mathrm{Oh}(13) \cdots \mathrm{O}(1)$ & $2.856(7)$ & $\mathrm{Ow}(17) \cdots \mathrm{O}(10)$ & $3.259(8)$ \\
\hline $\mathrm{Oh}(14) \cdots \mathrm{Ow}(19)$ & $2.610(8)$ & $\mathrm{Oh}(18) \cdots \mathrm{Ow}(19)$ & $3.423(8)$ \\
\hline $\mathrm{Oh}(15)$ & - & $\mathrm{Ow}(19) \cdots \mathrm{Oh}(14)$ & $2.610(8)$ \\
\hline $\mathrm{Oh}(16) \cdots \mathrm{O}(12)$ & $2.782(8)$ & & \\
\hline
\end{tabular}

provide increased resolution. Details of the intensity data collection of LAUB2 are given in Table 1. The data were processed with the Nonius program suite DENZO-SMN and corrected for Lorentz, polarization, background and absorption effects. For both LAUB1 and LAUB2, observed extinctions were in accordance with space groups
$P b c m$ and $P b c 2_{1}$, and intensity statistics gave very strong indications of the presence of a symmetry centre. The crystal structure was solved from the LAUB1 dataset by direct methods in space group Pbcm (SHELXS-97, Sheldrick, 1997a) and the positions of all $\mathrm{Fe}, \mathrm{P}$ and $\mathrm{O}$ atoms were found almost immediately. A full-matrix least-squares 


\section{U. KOLITSCH}

TABLE 5. Bond-valence analysis for kidwellite.

\begin{tabular}{|c|c|c|c|c|c|c|c|c|c|c|}
\hline & $\mathrm{Fe}(1)$ & $\mathrm{Fe}(2)$ & $\mathrm{Fe}(3)$ & $\mathrm{Fe}(4)$ & $\mathrm{Fe}(5)$ & $\mathrm{P}(1)$ & $\mathrm{P}(2)$ & $\mathrm{P}(3)$ & $\mathrm{Na}$ & Sum* \\
\hline $\mathrm{O}(1)$ & & & 0.512 & & & 1.298 & & & & 1.81 \\
\hline $\mathrm{O}(2)$ & & & & 0.542 & & 1.274 & & & & 1.82 \\
\hline $\mathrm{O}(3)$ & 0.393 & 0.501 & & & & 1.163 & & & & 2.06 \\
\hline $\mathrm{O}(4)$ & 0.370 & 0.369 & & & & 1.146 & & & & 1.89 \\
\hline $\mathrm{O}(5)$ & & 0.589 & & & & & 1.242 & & $\begin{array}{c}0.139 \\
\times 2\end{array}$ & 1.97 \\
\hline $\mathrm{O}(6)$ & & & & & $\begin{array}{c}0.510 \\
\times 2\end{array}$ & & 1.235 & & & 1.75 \\
\hline $\mathrm{O}(7)$ & & & & 0.587 & & & 1.232 & & & 1.82 \\
\hline $\mathrm{O}(8)$ & & 0.658 & & & & & 1.186 & & $\begin{array}{c}0.186 \\
\times 2\end{array}$ & 2.03 \\
\hline $\mathrm{O}(9)$ & & & & 0.618 & & & & 1.300 & & 1.92 \\
\hline $\mathrm{O}(10)$ & 0.715 & & & & & & & 1.262 & & 1.98 \\
\hline $\mathrm{O}(11)$ & & & 0.595 & & & & & 1.228 & & 1.82 \\
\hline $\mathrm{O}(12)$ & 0.681 & & & & & & & 1.182 & & 1.86 \\
\hline $\operatorname{Oh}(13)$ & 0.597 & & 0.546 & & & & & & & 1.14 \\
\hline $\operatorname{Oh}(14)$ & & 0.550 & & 0.430 & & & & & & 0.98 \\
\hline $\operatorname{Oh}(15)$ & 0.363 & 0.360 & & 0.386 & & & & & & 1.11 \\
\hline $\mathrm{Oh}(16)$ & & & $\begin{array}{l}0.490 \\
0.472\end{array}$ & & & & & & & 0.96 \\
\hline $\mathrm{Ow}(17)$ & & & 0.375 & & & & & & & 0.38 \\
\hline $\mathrm{Oh}(18)$ & & & & 0.482 & $\begin{array}{c}0.537 \\
\times 2\end{array}$ & & & & & 1.02 \\
\hline Ow(19) & & & & & $\begin{array}{c}0.463 \\
\times 2\end{array}$ & & & & $\begin{array}{c}0.245 \\
\times 2\end{array}$ & $0.71 * *$ \\
\hline Sum* & 3.12 & 3.03 & 2.99 & 3.05 & 3.02 & 4.88 & 4.90 & 4.97 & 1.14 & \\
\hline
\end{tabular}

Bond-valence parameters used are from Brese and O'Keeffe (1991).

* Sum values involving bonds to $\mathrm{P}(1), \mathrm{P}(2)$ and $\mathrm{P}(3)$ are based on the refined occupancies (P:As ratios) of these sites (see Table 2). Because of the very low occupancy and uncertain nature of the $M(1), M(2)$ and $M(3)$ sites, their bondvalence contributions have not been taken into account; however, their important influence is discussed in the text. ** Occupancy of Ow(19) site is probably close to $\left(\mathrm{OH}_{0.5} \mathrm{H}_{2} \mathrm{O}_{0.5}\right)$ (see text).

refinement on $F^{2}$ in space group $P b c m$ (SHELXL97, Sheldrick $1997 b$; see Table 1), involving anisotropic displacement parameters for all atoms, rapidly converged to $R 1 \sim 7 \%$. At this point, the Fourier difference map revealed one small but distinct, above-background peak representing a site [5+1]-coordinated by $\mathrm{O}$ atoms at distances between $\sim 1.9$ and $\sim 2.6 \AA$. This site, designated $M(1)$, was therefore assumed to be partially occupied by $\mathrm{Cu}$ and was modelled accordingly, while fixing its isotropic displacement parameter to a reasonable value; site occupancy refinements gave an occupancy factor of $\sim 3 \%$. The positions of $\mathrm{H}$ atoms could not be located. The refinement of LAUB1 converged to the final agreement factor $R 1=6.55 \%$. This comparatively high residual is due to the poor counting statistics and the imperfect nature of the crystal fragment measured. A structure refinement using the LAUB2 dataset confirmed the presence of the $M(1)$ site, and led to the detection of a second, similar site, designated $M(2)$. The refinement converged at $R 1=3.14 \%$ (Table 1 ). Positions of four $\mathrm{H}$ atoms could be located. By contrast with LAUB1, the refinement of LAUB2 demonstrated the presence of minor As on two P sites, in agreement with the chemical analyses. The maximum peaks in the final differenceFourier map were (LAUB1) 1.74 and $-0.93 \mathrm{e} / \AA^{3}$, and (LAUB2) 0.83 and $-0.84 \mathrm{e} / \AA^{3}$, respectively. The character of the strongest positive peaks is discussed below.

The final atomic positions and displacement parameters of LAUB2 are given in Tables 7 and 8. Selected bond lengths and calculated bond valences are listed in Table 9, including suggested 


\section{CRYSTAL STRUCTURES OF KIDWELLITE AND 'LAUBMANNITE'}

TABLE 7. Fractional atomic coordinates and isotropic displacement parameters for 'laubmannite' (Moore, 1970).

\begin{tabular}{|c|c|c|c|c|c|}
\hline Atom & $x$ & $y$ & $z$ & $U_{\text {eq }} / U_{\text {iso }}$ & Occupancy \\
\hline $\mathrm{Fe}(1)$ & $-0.74484(9)$ & $0.87846(4)$ & $0.611299(17)$ & $0.00747(14)$ & \\
\hline $\mathrm{Fe}(2)$ & $-0.75356(10)$ & $0.95118(4)$ & $0.699478(18)$ & $0.01148(15)$ & \\
\hline $\mathrm{Fe}(3)$ & $-0.00434(11)$ & $0.56070(4)$ & $0.542350(18)$ & $0.01121(15)$ & \\
\hline $\mathrm{Fe}(4)$ & $-0.00790(10)$ & $0.70285(4)$ & $0.689659(17)$ & $0.00993(15)$ & \\
\hline$M(1)$ & $0.516(4)$ & $0.7015(16)$ & $0.6889(7)$ & $0.02 * *$ & $0.0245(16)^{*}$ \\
\hline$M(2)$ & $-0.488(6)$ & $0.561(2)$ & $0.5416(10)$ & $0.02 * *$ & $0.0180(16)^{*}$ \\
\hline $\mathrm{P}(1)$ & $0.24076(14)$ & $0.52365(6)$ & $0.63956(2)$ & $0.0066(2)$ & $\mathrm{P}_{0.874(3)} \mathrm{As}_{0.126(3)}$ \\
\hline $\mathrm{P}(2)$ & $-0.23810(16)$ & $0.74415(7)$ & $0.59199(3)$ & $0.00513(19)$ & $\mathrm{P}_{1.00}$ \\
\hline $\mathrm{P}(3)$ & $-0.2554(2)$ & $0.86207(9)$ & 0.75 & $0.0065(3)$ & $\mathrm{P}_{0.952(3)} \mathrm{As}_{0.048(3)}$ \\
\hline $\mathrm{O}(1)$ & $0.2364(5)$ & $0.5553(2)$ & $0.59225(9)$ & $0.0122(6)$ & \\
\hline$O(2)$ & $0.2535(5)$ & $0.60714(19)$ & $0.67209(9)$ & $0.0112(5)$ & \\
\hline $\mathrm{O}(3)$ & $-0.4843(5)$ & $0.95979(18)$ & $0.64813(8)$ & $0.0096(5)$ & \\
\hline $\mathrm{O}(4)$ & $-0.9926(5)$ & $0.96279(18)$ & $0.64946(8)$ & $0.0099(5)$ & \\
\hline $\mathrm{O}(5)$ & $-0.5061(5)$ & $0.7886(2)$ & $0.58904(10)$ & $0.0171(6)$ & \\
\hline$O(6)$ & $-0.1905(6)$ & $0.6973(2)$ & $0.63559(9)$ & $0.0164(6)$ & \\
\hline $\mathrm{O}(7)$ & $-1.0379(5)$ & $0.8232(2)$ & $0.58340(9)$ & $0.0158(6)$ & \\
\hline $\mathrm{O}(8)$ & $-0.2213(5)$ & $0.66974(19)$ & $0.55583(9)$ & $0.0135(6)$ & \\
\hline $\mathrm{O}(9)$ & $-0.2568(5)$ & $0.80081(19)$ & $0.70931(9)$ & $0.0131(6)$ & \\
\hline $\mathrm{O}(10)$ & $-0.0135(7)$ & $0.9291(3)$ & 0.75 & $0.0154(8)$ & \\
\hline $\mathrm{O}(11)$ & $-0.5044(7)$ & $0.9259(3)$ & 0.75 & $0.0131(8)$ & \\
\hline $\mathrm{Oh}(12)$ & $-0.2748(5)$ & $0.4753(2)$ & $0.56640(9)$ & $0.0138(6)$ & \\
\hline $\mathrm{Oh}(13)$ & $0.2459(5)$ & $0.81073(19)$ & $0.67283(9)$ & $0.0101(5)$ & \\
\hline $\mathrm{Oh}(14)$ & $-0.2634(5)$ & $0.5946(2)$ & $0.70882(9)$ & $0.0125(6)$ & \\
\hline $\mathrm{Oh}(15)$ & $0.1705(6)$ & $0.4452(2)$ & $0.51631(9)$ & $0.0171(6)$ & \\
\hline Ow(16) & $0.2873(6)$ & $0.6475(2)$ & $0.51433(10)$ & $0.0224(7)$ & \\
\hline $\mathrm{Oh}(17)$ & $0.1355(7)$ & $0.6964(3)$ & 0.75 & $0.0123(8)$ & \\
\hline $\mathrm{H}(1)$ & $-0.415(7)$ & $0.508(4)$ & $0.5742(18)$ & $0.040(16)$ & \\
\hline $\mathrm{H}(2)$ & $0.411(5)$ & $0.797(5)$ & $0.676(2)$ & $0.07(2)$ & \\
\hline $\mathrm{H}(3)$ & $-0.413(7)$ & $0.607(5)$ & $0.6968(18)$ & $0.051(19)$ & \\
\hline $\mathrm{H}(4)$ & $0.128(14)$ & $0.399(4)$ & $0.5351(18)$ & $0.07(2)$ & \\
\hline
\end{tabular}

Oxygens of hydroxyl groups are designated as Oh, those of water molecules as Ow.

* Both $M(1)$ and $M(2)$ sites were refined as $\mathrm{Cu}$ (see text); their $U_{\text {iso }}$ values have been fixed.

** fixed

hydrogen bonds. A bond-valence analysis is presented in Table 10. A list of observed and calculated structure factors (Table 11) has been deposited with the Principal Editor of Mineralogical Magazine and is available upon request.

\section{Description and discussion of the structures}

\section{Kidwellite}

The structure solution demonstrates that kidwellite crystallizes in space group $P 2 / c$, and that the previously reported space group $(A 2 / m, A m$ or $A 2$; Moore and Ito, 1978) is incorrect. The space group revision may explain the statement of
Moore and Ito (1978) that "Although the $b$ - and $c$-axes could be established with confidence, selection of the $a$-axis presented serious problems". The observation of the presence of a medium-strong (010) reflection, violating $A$ centering, in the X-ray powder diffraction pattern of kidwellite (Moore, 1970; Walenta and Binder, 1980) is confirmed, and validated by a calculated powder pattern $\left(I / I_{100}=0.97\right)$. The structure model obtained was used by Kia Wallwork and Allan Pring (pers. comm.) to perform a Rietveld refinement of synchrotron powder diffraction data collected on a kidwellite sample from Australia. This sample is nearly pure (it contains only trace amounts of $\mathrm{Al}$, but no $\mathrm{Cu}$ or 


\section{U. KOLITSCH}

TABLE 8. Anisotropic displacement parameters for 'laubmannite' (Moore, 1970).

\begin{tabular}{lcccrrr}
\hline Atom & $U_{11}$ & $U_{22}$ & $U_{33}$ & $U_{23}$ & \multicolumn{1}{c}{$U_{13}$} & \multicolumn{1}{c}{$U_{12}$} \\
\hline $\mathrm{Fe}(1)$ & $0.0062(3)$ & $0.0065(3)$ & $0.0097(3)$ & $0.00004(19)$ & $-0.00009(19)$ & \multicolumn{1}{c}{$0.00087(18)$} \\
$\mathrm{Fe}(2)$ & $0.0112(3)$ & $0.0129(3)$ & $0.0103(3)$ & $-0.0008(2)$ & $-0.0008(2)$ & \multicolumn{1}{c}{$0.0000(2)$} \\
$\mathrm{Fe}(3)$ & $0.0107(3)$ & $0.0100(3)$ & $0.0130(3)$ & $0.0003(2)$ & $0.0012(2)$ & $0.0006(2)$ \\
$\mathrm{Fe}(4)$ & $0.0101(3)$ & $0.0095(3)$ & $0.0102(3)$ & $-0.0010(2)$ & $-0.0007(2)$ & $0.0022(2)$ \\
$\mathrm{P}(1)$ & $0.0051(4)$ & $0.0059(4)$ & $0.0088(4)$ & $-0.0004(3)$ & $0.0000(3)$ & \multicolumn{1}{c}{$0.0005(3)$} \\
$\mathrm{P}(2)$ & $0.0051(4)$ & $0.0039(4)$ & $0.0064(4)$ & $-0.0010(3)$ & $-0.0005(3)$ & $0.0011(3)$ \\
$\mathrm{P}(3)$ & $0.0044(6)$ & $0.0046(6)$ & $0.0105(6)$ & 0.0 & 0.0 & $0.0005(4)$ \\
$\mathrm{O}(1)$ & $0.0107(13)$ & $0.0155(14)$ & $0.0104(13)$ & $0.0002(11)$ & $-0.0025(10)$ & $-0.0012(10)$ \\
$\mathrm{O}(2)$ & $0.0119(13)$ & $0.0076(13)$ & $0.0141(13)$ & $-0.0041(11)$ & $-0.0003(10)$ & $0.0025(10)$ \\
$\mathrm{O}(3)$ & $0.0103(12)$ & $0.0050(12)$ & $0.0135(12)$ & $0.0015(10)$ & $-0.0017(10)$ & $-0.0034(10)$ \\
$\mathrm{O}(4)$ & $0.0077(12)$ & $0.0097(13)$ & $0.0122(12)$ & $-0.0005(10)$ & $-0.0008(10)$ & $0.0040(10)$ \\
$\mathrm{O}(5)$ & $0.0103(13)$ & $0.0180(15)$ & $0.0231(15)$ & $-0.0078(12)$ & $-0.0010(12)$ & $0.0048(11)$ \\
$\mathrm{O}(6)$ & $0.0222(14)$ & $0.0166(15)$ & $0.0105(13)$ & $0.0044(11)$ & $-0.0052(11)$ & $-0.0018(12)$ \\
$\mathrm{O}(7)$ & $0.0144(13)$ & $0.0158(14)$ & $0.0173(14)$ & $0.0023(11)$ & $-0.0028(11)$ & $-0.0067(11)$ \\
$\mathrm{O}(8)$ & $0.0190(14)$ & $0.0086(13)$ & $0.0129(13)$ & $-0.0047(11)$ & $-0.0015(11)$ & $0.0058(11)$ \\
$\mathrm{O}(9)$ & $0.0149(13)$ & $0.0111(14)$ & $0.0131(13)$ & $-0.0031(11)$ & $-0.0001(11)$ & $0.0054(11)$ \\
$\mathrm{O}(10)$ & $0.0127(18)$ & $0.0074(19)$ & $0.026(2)$ & 0.0 & 0.0 & $-0.0021(15)$ \\
$\mathrm{O}(11)$ & $0.0109(17)$ & $0.0102(19)$ & $0.0183(19)$ & 0.0 & 0.0 & $0.0019(15)$ \\
Oh(12) & $0.0126(13)$ & $0.0144(14)$ & $0.0144(13)$ & $0.0036(11)$ & $0.0033(11)$ & $0.0018(11)$ \\
Oh(13) & $0.0063(12)$ & $0.0096(13)$ & $0.0144(13)$ & $0.0039(10)$ & $-0.0002(10)$ & $0.0002(10)$ \\
Oh(14) & $0.0133(13)$ & $0.0118(14)$ & $0.0124(13)$ & $0.0002(11)$ & $0.0010(11)$ & $-0.0017(11)$ \\
Oh(15) & $0.0209(15)$ & $0.0162(15)$ & $0.0143(14)$ & $-0.0026(12)$ & $0.0024(12)$ & $-0.0021(12)$ \\
Ow(16) & $0.0203(15)$ & $0.0239(18)$ & $0.0229(16)$ & $0.0086(13)$ & $-0.0030(12)$ & $-0.0100(13)$ \\
Oh(17) & $0.0092(17)$ & $0.020(2)$ & $0.0079(17)$ & 0.000 & 0.0000 .000 & $0.0022(15)$ \\
& & & & & & \\
\hline & & & & & & \\
\hline
\end{tabular}

As, or other impurity elements) and has refined unit-cell parameters, $a=20.022, b=5.166, c=$ $13.983 \AA, \beta=106.867^{\circ}, V=1383.97 \AA^{3}$, which are slightly smaller than those of the slightly arsenatian sample studied here, the volume of which is $0.7 \%$ larger. The structure model determined here is confirmed in detail by the Rietveld refinement $\left(R_{\mathrm{wp}}=2.6 \%\right)$, although none of the metal-oxygen distances in the Australian sample could be refined without severe restraints.

Kidwellite has a complex framework structure which shows some close relations to that of 'laubmannite' (see below). Unlike the structure proposed by Moore (1970), kidwellite does not contain any ' $h$-cluster' (trimers of three facesharing $\left(\mathrm{Fe}^{2+}, \mathrm{Fe}^{3+}\right) \mathrm{O}_{6}$ octahedra). The structure contains (Table 2) five $\mathrm{Fe}$ atoms, one $\mathrm{Na}$ atom, three $\mathrm{P}$ atoms, $19 \mathrm{O}$ atoms, about $19 \mathrm{H}$ atoms (none of which were detectable during the refinement), and three additional, cation sites with very low occupancies (see below). The Fe atoms are all [6]-coordinated by $\mathrm{O}$ atoms to form somewhat distorted $\mathrm{FeO}_{6}$ octahedra. These octahedra have very similar average $\mathrm{Fe}-\mathrm{O}$ bond lengths which range from 2.014 to $2.022 \AA$, and bond-valence calculations demonstrate that all $\mathrm{Fe}$ sites host trivalent Fe (Table 5). The very minor Al contents detected by SEM-EDS seem to be homogeneously distributed among all five $\mathrm{Fe}$ sites.

The $\mathrm{P}$ atom in each of the three $\mathrm{PO}_{4}$ tetrahedra is partially substituted by As atoms. In the $\mathrm{P}(1) \mathrm{O}_{4}$ group the P:As ratio is $\sim 86: 14$, whereas in the $\mathrm{P}(2) \mathrm{O}_{4}$ and $\mathrm{P}(3) \mathrm{O}_{4}$ groups, the ratios are distinctly larger, 94:6 and 97:3, respectively (Table 2). Average $\mathrm{P}-\mathrm{O}$ bond lengths for $\mathrm{P}(1), \mathrm{P}(2)$ and $\mathrm{P}(3)$ are $1.559,1.542$ and $1.530 \AA$ (Table 4), respectively, and are consistent with the refined P:As ratios.

Dominant elements of the kidwellite structure comprise three different, $\mathrm{FeO}_{6}$-based polyhedral clusters (Fig. 1): one dimer composed of two face-sharing $\mathrm{Fe}(1) \mathrm{O}_{6}$ and $\mathrm{Fe}(2) \mathrm{O}_{6}$ octahedra, another built of two edge-sharing $\mathrm{Fe}(3) \mathrm{O}_{6}$ octahedra and one trimer built of three cornersharing $\mathrm{FeO}_{6}$ octahedra $\left(\mathrm{Fe}(4) \mathrm{O}_{6}-\mathrm{Fe}(5) \mathrm{O}_{6}-\right.$ $\left.\mathrm{Fe}(4) \mathrm{O}_{6}\right)$. These dimers and trimer are cornerlinked to neighbouring, symmetry-related units, and to $\mathrm{PO}_{4}$ tetrahedra. The resulting threedimensional framework hosts narrow channels 


\section{CRYSTAL STRUCTURES OF KIDWELLITE AND 'LAUBMANNITE'}

TABLE 9. Selected bond distances $(\AA)$ for the coordination polyhedra in 'laubmannite' (Moore, 1970).

\begin{tabular}{|c|c|c|c|}
\hline $\mathrm{Fe}(1)-\mathrm{O}(5)$ & $1.897(3)$ & $\mathrm{P}(1)-\mathrm{O}(1)$ & $1.536(3)$ \\
\hline$-O(7)$ & $1.911(3)$ & $-\mathrm{O}(2)$ & $1.547(3)$ \\
\hline$-\mathrm{O}(12)$ & $1.947(3)$ & $-\mathrm{O}(3)$ & $1.567(3)$ \\
\hline$-\mathrm{O}(3)$ & $2.102(3)$ & $-\mathrm{O}(4)$ & $1.570(3)$ \\
\hline$-\mathrm{O}(4)$ & $2.109(3)$ & $<\mathrm{P}(1)-\mathrm{O}>$ & 1.555 \\
\hline$-\mathrm{O}(13)$ & $2.132(4)$ & & \\
\hline \multirow{2}{*}{$<\mathrm{Fe}(1)-\mathrm{O}>$} & 2.016 & $\mathrm{P}(2)-\mathrm{O}(5)$ & $1.521(3)$ \\
\hline & & $-\mathrm{O}(6)$ & $1.524(3)$ \\
\hline $\mathrm{Fe}(2)-\mathrm{O}(4)$ & $1.993(3)$ & $-\mathrm{O}(8)$ & $1.534(3)$ \\
\hline$-\mathrm{O}(14)$ & $2.029(3)$ & $-O(7)$ & $1.539(3)$ \\
\hline$-\mathrm{O}(11)$ & $2.063(3)$ & $<\mathrm{P}(2)-\mathrm{O}>$ & 1.53 \\
\hline$-\mathrm{O}(10)$ & 2.091(3) & & \\
\hline$-\mathrm{O}(3)$ & $2.123(3)$ & $\mathrm{P}(3)-\mathrm{O}(9) \times 2$ & $1.528(3)$ \\
\hline$-\mathrm{O}(13)$ & $2.132(4)$ & $-\mathrm{O}(10)$ & $1.565(4)$ \\
\hline \multirow[t]{2}{*}{$<\mathrm{Fe}(2)-\mathrm{O}>$} & 2.072 & $-\mathrm{O}(11)$ & $1.568(4)$ \\
\hline & & $<\mathrm{P}(3)-\mathrm{O}>$ & 1.547 \\
\hline $\mathrm{Fe}(3)-\mathrm{O}(8)$ & $1.942(3)$ & & \\
\hline$-\mathrm{O}(12)$ & $1.987(3)$ & $M(1)-\mathrm{O}(9)$ & $1.89(3)$ \\
\hline$-\mathrm{O}(1)$ & $1.990(3)$ & $-\mathrm{O}(14)$ & $1.99(3)$ \\
\hline$-\mathrm{O}(15)$ & $2.014(3)$ & $-\mathrm{O}(2)$ & $2.00(3)$ \\
\hline$-\mathrm{O}(15)$ & $2.021(3)$ & $-\mathrm{O}(13)$ & $2.13(3)$ \\
\hline$-\mathrm{O}(16)$ & $2.121(3)$ & $-\mathrm{O}(6)$ & $2.23(3)$ \\
\hline \multirow[t]{2}{*}{$<\mathrm{Fe}(3)-\mathrm{O}>$} & 2.013 & $-\mathrm{O}(17)$ & $2.76(3)$ \\
\hline & & $<M(1)-\mathrm{O}>$ & 2.17 \\
\hline $\mathrm{Fe}(4)-\mathrm{O}(6)$ & $1.931(3)$ & & \\
\hline$-\mathrm{O}(9)$ & $1.977(3)$ & $M(2)-\mathrm{O}(12)$ & $1.80(3)$ \\
\hline$-\mathrm{O}(2)$ & $1.980(3)$ & $-\mathrm{O}(16)$ & $1.88(3)$ \\
\hline$-\mathrm{O}(17)$ & $2.0190(17)$ & $-\mathrm{O}(8)$ & $2.11(3)$ \\
\hline$-\mathrm{O}(13)$ & $2.066(4)$ & $-\mathrm{O}(1)$ & $2.13(3)$ \\
\hline$-\mathrm{O}(14)$ & $2.096(3)$ & $-\mathrm{O}(15)$ & $2.44(3)$ \\
\hline \multirow[t]{2}{*}{$<\mathrm{Fe}(4)-\mathrm{O}>$} & 2.012 & $-\mathrm{O}(15)$ & $2.52(3)$ \\
\hline & & $<M(2)-\mathrm{O}>$ & 2.15 \\
\hline \multicolumn{4}{|c|}{ Possible hydrogen bonds* } \\
\hline $\mathrm{Oh}(12)-\mathrm{H}(1)^{* *}$ & $0.90(2)$ & $\mathrm{Oh}(12) \cdots \mathrm{O}(1)$ & $2.88(4)$ \\
\hline $\mathrm{Oh}(13)-\mathrm{H}(2)^{* *}$ & $0.88(2)$ & $\mathrm{Oh}(13) \cdots \mathrm{O}(9)$ & $2.81(4)$ \\
\hline $\mathrm{Oh}(14)-\mathrm{H}(3)^{* *}$ & $0.88(2)$ & $\mathrm{Oh}(14) \cdots \mathrm{O}(2)$ & $2.75(4)$ \\
\hline \multirow[t]{5}{*}{$\mathrm{Oh}(15)-\mathrm{H}(4)^{* *}$} & $0.89(2)$ & $\mathrm{Oh}(15) \cdots \mathrm{O}(7)$ & $2.78(4)$ \\
\hline & & $\mathrm{Oh}(12)-\mathrm{H}(1) \cdots \mathrm{O}(1)$ & $168(1)$ \\
\hline & & $\mathrm{Oh}(13)-\mathrm{H}(2) \cdots \mathrm{O}(9)$ & 153(1) \\
\hline & & $\mathrm{Oh}(14)-\mathrm{H}(3) \cdots \mathrm{O}(2)$ & $168(1)$ \\
\hline & & $\mathrm{Oh}(15)-\mathrm{H}(4) \cdots \mathrm{O}(7)$ & $167(1)$ \\
\hline
\end{tabular}

* $\mathrm{H}$ atoms belonging to $\mathrm{Ow}(16)$ and $\mathrm{Oh}(17)$ were not detectable, but possible hydrogen bonds are: $\mathrm{Ow}(16) \cdots \mathrm{O}(8)=2.87 \AA$, and $\mathrm{Oh}(17) \cdots \mathrm{O}(14)=2.82 \AA$.

** Restrained to distance of $0.90(2) \AA$

which run parallel to the principal axes (Fig. 1). The $\mathrm{Na}$ atom is located in the largest channel and shows a [6]-coordination sphere, in which the six $\mathrm{O}$ ligands form a slightly distorted (flattened) octahedron (Fig. 1), with an average $\mathrm{Na}-\mathrm{O}$ bond length of $2.424 \AA$ (Table 4) and a calculated bond-valence sum of 1.14 valence units (v.u.)
(Table 5). The anisotropic displacement parameters of the $\mathrm{Na}$ atom demonstrates some positional disorder parallel to the $5.19 \AA$ axis (Table 3). Occupancy refinements of the Na site indicated the weak possibility of a partially occupied site. However, the strong correlation between site occupancy and displacement para- 


\section{U. KOLITSCH}

TABLE 10. Bond-valence analysis for 'laubmannite' (Moore, 1970).

\begin{tabular}{|c|c|c|c|c|c|c|c|c|}
\hline & $\mathrm{Fe}(1)$ & $\mathrm{Fe}(2)$ & $\mathrm{Fe}(3)$ & $\mathrm{Fe}(4)$ & $\mathrm{P}(1)$ & $\mathrm{P}(2)$ & $\mathrm{P}(3)$ & Sum* \\
\hline $\mathrm{O}(1)$ & & & 0.536 & & 1.282 & & & 1.82 \\
\hline $\mathrm{O}(2)$ & & & & 0.550 & 1.244 & & & 1.79 \\
\hline $\mathrm{O}(3)$ & 0.396 & 0.362 & & & 1.178 & & & 1.94 \\
\hline $\mathrm{O}(4)$ & 0.388 & 0.514 & & & 1.169 & & & 2.07 \\
\hline $\mathrm{O}(5)$ & 0.689 & & & & & 1.251 & & 1.94 \\
\hline $\mathrm{O}(6)$ & & & & 0.628 & & 1.241 & & 1.87 \\
\hline $\mathrm{O}(7)$ & 0.663 & & & & & 1.192 & & 1.86 \\
\hline $\mathrm{O}(8)$ & & & 0.610 & & & 1.208 & & 1.82 \\
\hline $\mathrm{O}(9)$ & & & & 0.555 & & & $\begin{array}{c}1.269 \\
\times 2\end{array}$ & 1.82 \\
\hline $\mathrm{O}(10)$ & & $0.395 \times 2$ & & & & & 1.148 & 1.94 \\
\hline $\mathrm{O}(11)$ & & $0.426 \times 2$ & & & & & 1.139 & 1.99 \\
\hline $\mathrm{Oh}(12)$ & 0.602 & & 0.540 & & & & & 1.14 \\
\hline $\mathrm{Oh}(13)$ & 0.365 & 0.353 & & 0.436 & & & & 1.15 \\
\hline Oh(14) & & 0.467 & & 0.402 & & & & 0.87 \\
\hline \multirow[t]{2}{*}{$\mathrm{Oh}(15)$} & & & 0.502 & & & & & \\
\hline & & & 0.493 & & & & & 1.00 \\
\hline Ow(16) & & & 0.376 & & & & & 0.38 \\
\hline $\mathrm{Oh}(17)$ & & & & $0.495 \times 2$ & & & & 0.99 \\
\hline Sum* & 3.10 & 2.52 & 3.06 & 3.07 & 4.87 & 4.89 & 4.83 & \\
\hline
\end{tabular}

Bond-valence parameters used are from Brese and O'Keeffe (1991).

* Sum values involving bonds to $\mathrm{P}(1)$ and $\mathrm{P}(3)$ are based on the refined occupancies (P:As ratios) of these sites (see Table 6), and sum values involving bonds to $\mathrm{Fe}(2)$ are based on an assumed $\mathrm{Fe}^{2+}: \mathrm{Fe}^{3+}$ ratio of $1: 1$ on this site (see text; using only the parameters for either $\mathrm{Fe}^{3+}$ or $\mathrm{Fe}^{2+}$, one would obtain bond-valence sums of 2.60 and 2.43 v.u., respectively.) Because of the very low occupancy and uncertain nature of the two $M$ sites, their bond-valence contributions have not been taken into acccount; however, their important influence is discussed in the text.

Contributions of $\mathrm{H}$ atoms are not listed.

meters precluded any definite statement, and the occupancy has, therefore, been kept at $100 \%$. The wet-chemical analysis of type kidwellite (Moore and Ito, 1978) yielded $\mathrm{Na}$ contents which would indicate an occupancy of $\sim \mathrm{Na}_{0.71}$ for the $\mathrm{Na}$ site. However, the formula of the kidwellite from Broken Hill analysed by electron microprobe (Birch, 1990) corresponds to an occupancy of $\left(\mathrm{Na}_{0.9} \mathrm{Ca}_{0.05}\right)_{\Sigma 0.95}$ for the $\mathrm{Na}$ site.

Three further cation sites, $M(1), M(2)$ and $M(3)$, with very low occupancies and strongly distorted 'octahedral' coordinations with $M-\mathrm{O}$ bond lengths between $1.795(6)$ and $2.719(14) \AA$ (Table 4) are located in the channels of the framework (Fig. 2). On the basis of the geometries of these sites, refinement behaviour of their isotropic displacement factors during trial refinements and the fact that chemical analyses has shown $\mathrm{Cu}$ as an impurity in the crystals, it was assumed that $\mathrm{Cu}$ occupies, at least partly, these sites. Refined occupancies are basically identical: $\sim 7 \%$ for $M(1), \sim 7 \%$ for $M(2)$ and $\sim 7 \%$ for $M(3)$
(Table 2). The assignment of $\mathrm{Cu}$ to these sites is hypothetical, and other candidates could be Fe, trace amounts of $\mathrm{Mn}$, as well as $\mathrm{Na}$ atoms or $\mathrm{H}_{2} \mathrm{O}$ molecules. The Rietveld refinement, mentioned already, of a $\mathrm{Cu}$-free kidwellite sample by Kia Wallwork and Allan Pring (pers. comm.) also indicated very low occupancies of the $M$ sites. Therefore, $\mathrm{Cu}$ does not seem to be essential for the kidwellite structure.

The three $M$ sites in the crystal studied here have some fairly close contacts to several Fe sites: $M(1)-\mathrm{Fe}(5)=2.5925(5)(\times 2), M(2)-\mathrm{Fe}(3)=$ $2.526(16)$ and $2.660(16)$, and $M(3)-\mathrm{Fe}(4)=$ 2.442(13) and 2.744(13) ̊. Thus, repulsion forces preclude the possibility of high occupancies, and the neighbouring Fe sites might be locally vacant, if the respective $M$ site is occupied. An analysis of the bond-valence contributions of the three $M$ sites demonstrates that all those $\mathrm{O}$ atoms which are slightly underbonded (e.g. $\mathrm{O}(1), \mathrm{O}(2), \mathrm{O}(6)$ and $\mathrm{O}(7)$; Table 5) receive small bond-valence contribu- 


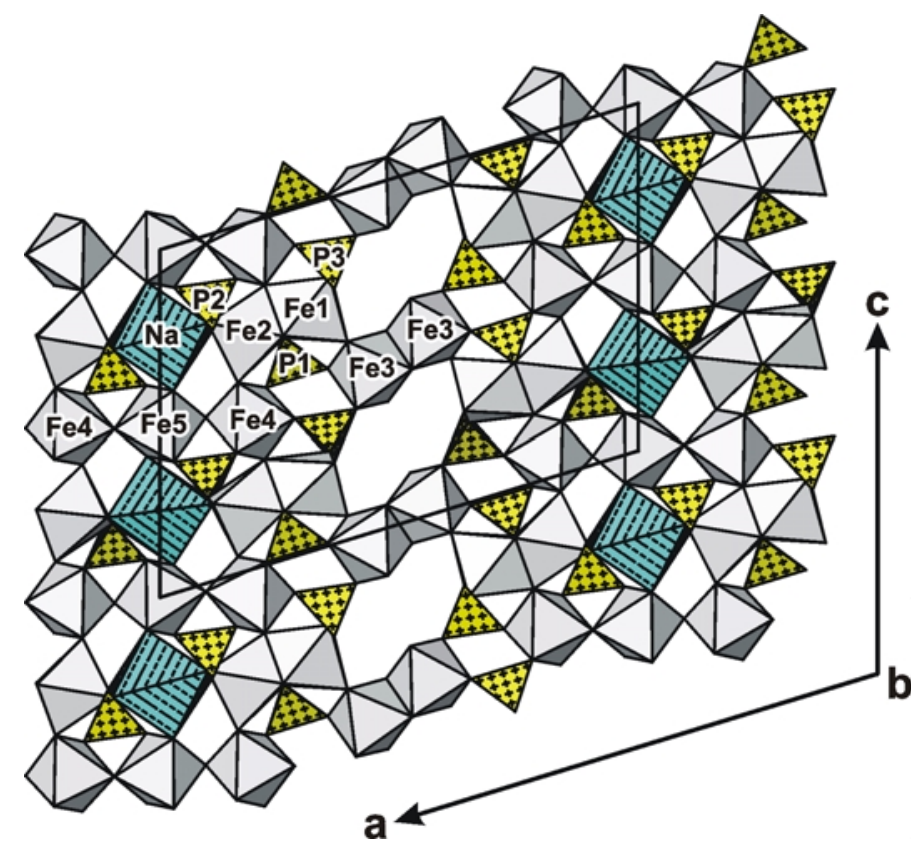

FIG. 1. The crystal structure of kidwellite projected along [010], i.e. the short $\sim 5.1 \AA$ axis. The $\mathrm{FeO}_{6}$-based building units (face-sharing dimer, edge-sharing dimer and corner-sharing trimer) are easily recognized in this view. The $\mathrm{NaO}_{6}$ octahedron (blue) is striped, and $\mathrm{PO}_{4}$ tetrahedra (yellow) are marked with crosses. The partially occupied sites $M(1), M(2)$ and $M(3)$ have been omitted for clarity. All drawings were done using ATOMS (Shape Software, 1999).

tions from the three $M$ sites (Table 4). By contrast, the $\mathrm{O}$ atoms which are more or less saturated (e.g. $\mathrm{O}(3), \mathrm{O}(5), \mathrm{O}(8)$; Table 5), do not receive any of these bond-valence contributions. Thus, the three $M$ sites provide a weak reinforcement of the atomic arrangement (cf. Fig. 2).

Derivation of the accurate chemical formula of kidwellite posed some problems. From the bondvalence calculations (Table 5) it is clear that $\mathrm{O}(1)$ to $\mathrm{O}(12)$ all represent $\mathrm{O}^{2-}$ anions, and that $\mathrm{Oh}(13)$ to $\mathrm{Oh}(16)$, and $\mathrm{Oh}(18)$ represent $\mathrm{OH}^{-}$groups. Ow(17) (0.38 v.u.) clearly forms part of a water molecule bonded to $\mathrm{Fe}(3)$ only, but $\mathrm{Ow}(19)$ (0.71 v.u.), which is bonded to $\mathrm{Fe}(5)$ and $\mathrm{Na}$, might represent a mixture of $\mathrm{H}_{2} \mathrm{O}$ and $\mathrm{OH}^{-}$. The condition of electroneutrality shows that the three partially $M$ sites are necessary for an accurate definition of a charge-balanced structure. If Ow(19) is assumed to represent only $\mathrm{H}_{2} \mathrm{O}$, then one obtains the detailed structural formula $\mathrm{NaFe}_{9}^{3+} M_{5 x}(\mathrm{OH})_{10}\left(\mathrm{H}_{2} \mathrm{O}\right)_{4}\left(\mathrm{PO}_{4}\right)_{6}$, where $M=$ $\mathrm{Fe}^{3+}, \mathrm{Cu}^{2+}$ or other metal cation, and $x \approx 0.07$. One formula unit then has $\sim 29$ positive charges (28.72 if $M=$ only $\mathrm{Cu}^{2+}$, or 29.08 if $M=$ only
$\mathrm{Fe}^{3+}$ ) and 28 negative charges. If, on the other hand, $\mathrm{Ow}(19)$ is assumed to have the occupancy $\left(\mathrm{OH}_{0.5} \mathrm{H}_{2} \mathrm{O}_{0.5}\right)$, the combined charge of all three $M$ sites is close to the value necessary for compensating the then 29 negative charges. This would correspond to the structural formula $\mathrm{NaFe}_{9}^{3+} M_{5 x}(\mathrm{OH})_{10}\left(\mathrm{OH}_{0.5} \mathrm{H}_{2} \mathrm{O}_{0.5}\right)_{2}\left(\mathrm{H}_{2} \mathrm{O}\right)_{2}\left(\mathrm{PO}_{4}\right)_{6}$. If $\mathrm{Ow}(19)$ is assumed to represent only $\mathrm{OH}$, then o n e obtains the formula $\mathrm{NaFe}_{9}^{3+} M_{5 x}(\mathrm{OH})_{12}\left(\mathrm{H}_{2} \mathrm{O}\right)_{2}\left(\mathrm{PO}_{4}\right)_{6}$, with 30 negative charges. As mentioned above, the refinement indicated the possibility of a partially occupied $\mathrm{Na}$ site. If confirmed, this would complicate matters further, but would also fit into the picture of an overall non-stoichiometric formula. The suggested simplified formula of kidwellite is therefore $\mathrm{Na}\left(\mathrm{Fe}^{3+}, M\right)_{9+x}(\mathrm{OH})_{11}\left(\mathrm{H}_{2} \mathrm{O}\right)_{3}\left(\mathrm{PO}_{4}\right)_{6}$, where $M=\mathrm{Fe}^{3+}, \mathrm{Cu}^{2+}$ or other metal cation, and $x \approx 0.3$. This formula also reflects the fact that the mineral contains no zeolitic ('free') water. As no $\mathrm{H}$ atoms could be detected in the refinement, the suggested hydrogen bonds (Table 4) are only tentative. Nonetheless, the suggested donoracceptor distances indicate that the hydrogen bonds are seemingly rather weak, with the 
exception of the short bond $\mathrm{Oh}(14) \cdots \mathrm{Ow}(19)$, 2.610(8) ^.

\section{'Laubmannite' and its relation to kidwellite}

The structure solution shows that 'laubmannite' as defined by Moore (1970) is a valid, but presently unnamed species which clearly deserves a species name. The space group $\mathrm{Pbcm}$ suggested by Moore (1970) is confirmed, although the formula had to be completely revised (see below).

'Laubmannite' has a complex framework structure comprising four $\mathrm{Fe}$ atoms, three $\mathrm{P}$ atoms, $17 \mathrm{O}$ atoms, $\sim 13 \mathrm{H}$ atoms (four of which were detectable during the refinement of LAUB2), and two cation sites, $M(1)$ and $M(2)$, with very low occupancies (Table 7). As in

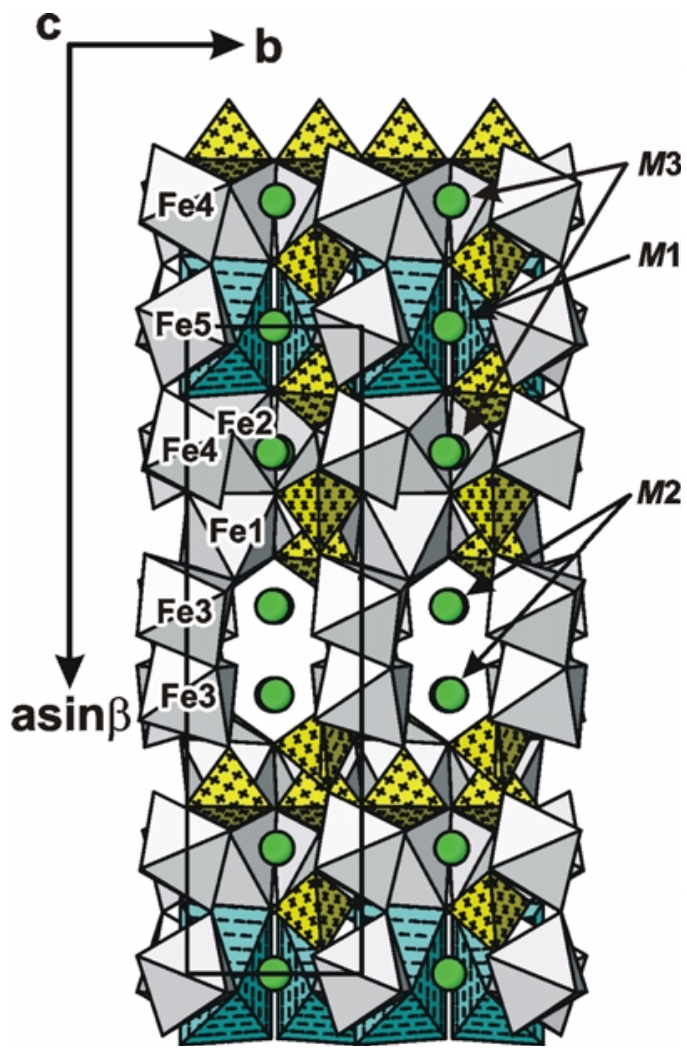

FIG. 2. View of the crystal structure of kidwellite along [001], showing the partially occupied sites $M(1), M(2)$ and $M(3)$ (green spheres), all of which are located in channels of the framework, and have strongly distorted 'octahedral' coordinations. The important role of these sites is discussed in the text. Legend otherwise as for

Fig. 1. Compare Fig. 4. kidwellite, all four $\mathrm{Fe}$ atoms are [6]-coordinated by $\mathrm{O}$ atoms to form distorted $\mathrm{FeO}_{6}$ octahedra (Fig. 3). The main building units are a dimer composed of two face-sharing $\mathrm{Fe}(1) \mathrm{O}_{6}$ and $\mathrm{Fe}(2) \mathrm{O}_{6}$ octahedra, a dimer composed of two edge-sharing $\mathrm{Fe}(3) \mathrm{O}_{6}$ octahedra and a dimer composed of two corner-sharing $\mathrm{Fe}(4) \mathrm{O}_{6}$ octahedra. Both $\mathrm{Fe}(1) / \mathrm{Fe}(2)$ - and $\mathrm{Fe}(3)$-based dimers also occur in kidwellite, whereas the $\mathrm{Fe}(4)$-based dimer contrasts with the $\mathrm{Fe}(4) / \mathrm{Fe}(5) / \mathrm{Fe}(4)$-based trimer of kidwellite. Two equivalent face-sharing $\mathrm{Fe}(1) \mathrm{O}_{6}-\mathrm{Fe}(2) \mathrm{O}_{6}$ dimers are connected via one common terminal edge to form a unique tetramer extended parallel to the $c$ axis (Figs 3,4).

Linkage between the $\mathrm{FeO}_{6}$-based building units is achieved by corner-sharing with adjacent $\mathrm{FeO}_{6}$ octahedra and by corner-linkage to $\mathrm{PO}_{4}$ tetrahedra

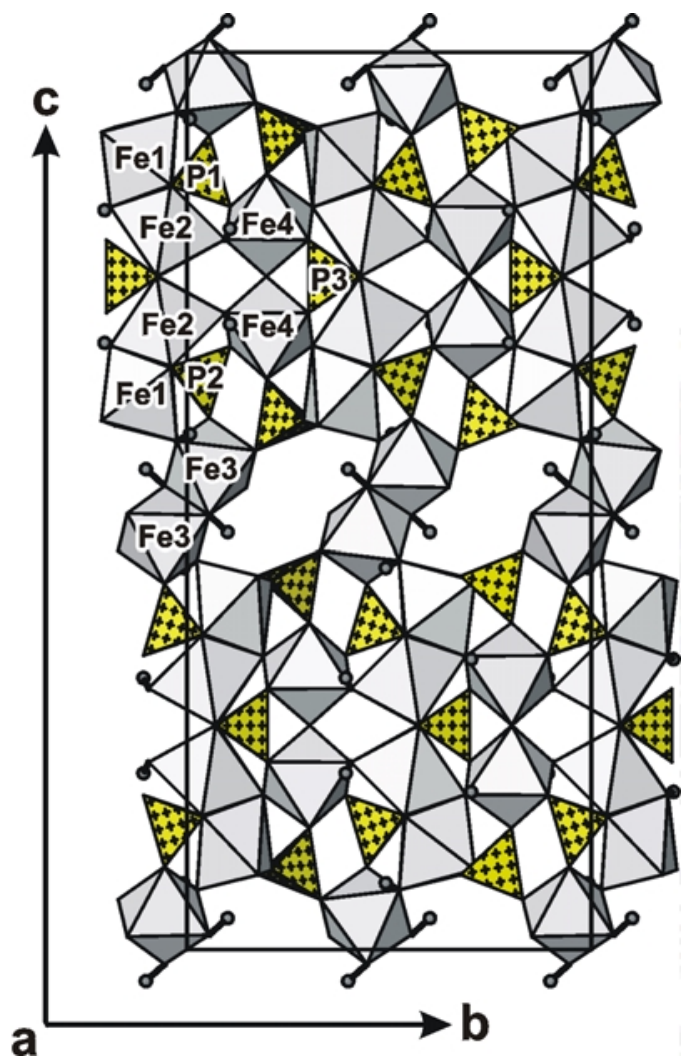

FIG. 3. The crystal structure of 'laubmannite' (Moore, 1970) projected along [100], i.e. the short $~ 5.1 \AA$ axis. The $\mathrm{FeO}_{6}$-based building units (face-sharing dimer, edge-sharing dimer and corner-sharing dimer) are easily recognized in this view (compare Figs 1 and 5). $\mathrm{O}-\mathrm{H}$ bonds are drawn as thin lines. Legend otherwise as for Fig. 1. 


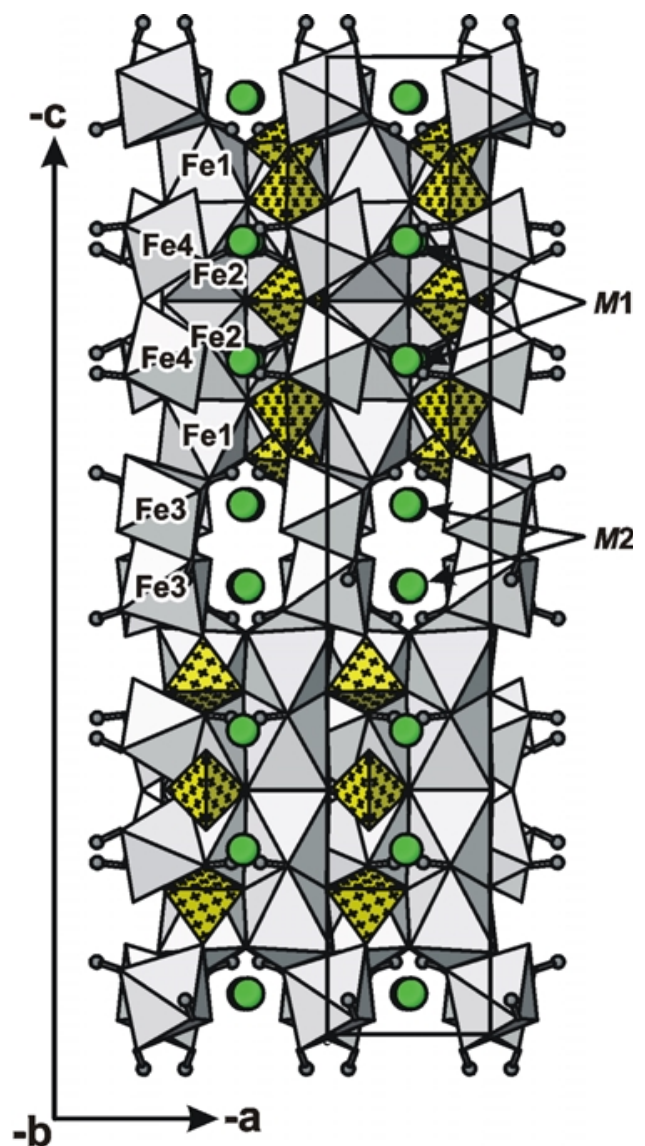

FIG. 4. View of the crystal structure of 'laubmannite' along [010], showing the partially occupied sites $M(1)$ and $M(2)$ (green spheres). Both sites are located in channels of the framework, and have strongly distorted [5+1] $[M(1)]$ and 'octahedral'

$[M(2)]$ coordinations. Compare also with Fig. 2.

(Figs 3,4). The $\mathrm{P}(1)$ site contains $\sim 13 \%$ As substituting for $\mathrm{P}$, the $\mathrm{P}(3)$ site $\sim 5 \%$ As, whereas the $\mathrm{P}(2)$ site is basically As-free (Table 7). As a result, the average $\mathrm{P}-\mathrm{O}$ bond lengths in the $\mathrm{P}(1)$-, $\mathrm{P}(2)$ - and $\mathrm{P}(3) \mathrm{O}_{4}$ tetrahedra are $1.555,1.530$ and $1.547 \AA$, respectively (Table 9). In the As-free LAUB1 the corresponding value of the average $\mathrm{P}(1)-\mathrm{O}$ bond length is, as expected, considerably lower: $1.542 \AA$. The $\mathrm{PO}_{4}$ tetrahedra show different degrees of geometrical distortion, which, as a comparison between LAUB2 and LAUB1 shows, is unrelated to the As content of LAUB2. The $\mathrm{P}(2) \mathrm{O}_{4}$ tetrahedron is the least distorted ( $\mathrm{P}-\mathrm{O}$ range: $1.521(3)$ to $1.539(3) \AA$ ). Both the $\mathrm{P}(1) \mathrm{O}_{4}$ and $\mathrm{P}(3) \mathrm{O}_{4}$ tetrahedra show considerable distortions, with bond length ranges of 1.536(3) to 1.570(3) $\AA$ for $\mathrm{P}(1)$, and 1.528(3) to $1.568(4) \AA$ for $\mathrm{P}(3)$, respectively. Again, this is in accordance with the observed As-for-P substitution on these two sites.

Average $\mathrm{Fe}-\mathrm{O}$ bond lengths for $\mathrm{Fe}(1)$ to $\mathrm{Fe}(4)$ are 2.016, 2.072, 2.013 and $2.012 \AA$, respectively. Bond-valence calculations indicate that $\mathrm{Fe}(1)$, $\mathrm{Fe}(3)$ and $\mathrm{Fe}(4)$ are occupied by $\mathrm{Fe}^{3+}$ only, whereas the average $\mathrm{Fe}$ valency of $\mathrm{Fe}(2)$ is $\sim 2.5+$ (Table 10). The average $\mathrm{Fe}(2)-\mathrm{O}$ distance, $2.072 \AA$ (Table 9), also provides evidence that $\mathrm{Fe}(2)$ is occupied by a mixture of ferrous and ferric iron in an approximate ratio of 1:1. According to Baur (1981), average $\mathrm{Fe}^{2+}-\mathrm{O}$ and $\mathrm{Fe}^{3+}-\mathrm{O}$ bond lengths in oxide iron compounds are 2.138 and $2.011 \AA$, respectively. The length of an 'ideal' $\left(\mathrm{Fe}_{0.5}^{2+} \mathrm{Fe}_{0.5}^{3+}\right)-\mathrm{O}$ bond, $2.075 \AA$, is indeed very close to the average $\mathrm{Fe}(2)-\mathrm{O}$ bond length in 'laubmannite'.

The relatively short $\mathrm{Fe}(1)-\mathrm{Fe}(2)$ distance in the face-sharing dimer, 2.924(1) $\AA$, makes it understandable that only this dimer accepts $\mathrm{Fe}^{2+}$, but not the other, edge- and corner-sharing dimers: if it would host only $\mathrm{Fe}^{3+}$ cations, electrostatic cation repulsion across the shared face would be much stronger and could lead to severe intrapolyhedral strain. In kidwellite, which contains only $\mathrm{Fe}^{3+}$ ions in an otherwise very similar face-sharing dimer, the $\mathrm{Fe}(1)-\mathrm{Fe}(2)$ distance is considerably longer, 2.967(2) $\AA$, as would be expected from a stronger repulsion across the shared face. The dark greenish to greenish brown colour and the strong pleochroism of 'laubmannite' is due to $\mathrm{Fe}^{2+}-\mathrm{Fe}^{3+}$ charge transfer within the face-, edge- and cornersharing dimeric units, typical of ferric-ferrous iron phosphates (Moore, 1970).

The three-dimensional framework of 'laubmannite' hosts narrow channels parallel to the $a$ and $b$ axes, as in kidwellite. In one of these channels, a further cation site, $M(1)$, with a very low occupancy $(\sim 3 \%$, if refined as $\mathrm{Cu})$ and a strongly distorted [5+1]-coordination is located in a void between two $\mathrm{Fe}(4) \mathrm{O}_{6}$ octahedra (Fig. 4, Table 9). A second cation site, $M(2)$, with an equally low occupancy $(\sim 2 \%)$ but a distorted 'octahedral' coordination, is present in a different channel (Fig. 4, Table 9). As in kidwellite, both $\mathrm{Cu}^{2+}$, $\mathrm{Fe}^{3+}$ and other minor metal cations might be present on these two sites. The $M(1)$ site has two fairly short distances to $\mathrm{Fe}(4), 2.44(3)$ and 2.74(3) A. Similarly, the $M(2)$ site has fairly short distances to $\mathrm{Fe}(3)$. Thus, repulsive forces 
will preclude any high occupancy of the two $M$ sites, comparable to the situation of the three partially occupied $M$ sites in kidwellite. If one takes the $M(1)$ site into account for the description of the framework connectivity, chains of facesharing, alternating $\mathrm{Fe}(M(1)) \mathrm{O}_{6}$ octahedra running along the $\sim 5.1 \AA$ axis ( $a$-axis) would result. Similar iron-deficient octahedral chains are known in lipscombite, $\left(\mathrm{Fe}^{2+}, \mathrm{Mn}^{2+}\right) \mathrm{Fe}_{2}^{3+}$ $\left(\mathrm{PO}_{4}\right)_{2}(\mathrm{OH})_{2}$ (Vencato et al., 1989). Partial occupancies of $\mathrm{Fe}$ sites have also been encountered in other iron phosphates: examples include rockbridgeite (Moore, 1970), whitmoreite (Moore et al., 1974), and synthetic $\mathrm{Fe}^{2+} \mathrm{Fe}_{2}^{3+}\left(\mathrm{PO}_{3} \mathrm{OH}\right)_{4}$ $\left(\mathrm{H}_{2} \mathrm{O}\right)_{4}$ (Vencato et al., 1986). As in kidwellite, all those $\mathrm{O}$ atoms which are most underbonded $(\mathrm{O}(1), \mathrm{O}(2), \mathrm{O}(8), \mathrm{O}(9)$; Table 10) receive small bond-valence contributions from the two $M$ sites (Table 9).

The chemical formula of 'laubmannite' obtained from the structure model is distinctly different from that suggested by Moore (1970). Judging from the bond-valence sums of the $\mathrm{O}$ atoms, five of them represent $\mathrm{OH}$ groups $[\mathrm{Oh}(12)$, $\mathrm{Oh}(13), \mathrm{Oh}(14), \mathrm{Oh}(15)$ and $\mathrm{Oh}(17)]$, whereas $\mathrm{Ow}(16)$, with only 0.38 v.u., is part of a $\mathrm{H}_{2} \mathrm{O}$ molecule (Tables 9, 10). If one also takes into account the occupancy of the $\mathrm{Fe}(2)$ site $\left(\mathrm{Fe}^{3+}: \mathrm{Fe}^{2+}\right.$ $\approx 1: 1$, and the two partially occupied $M$ sites, the accurate structural formula is $\left(\mathrm{Fe}^{3+}, \mathrm{Fe}^{2+}\right)_{2} \mathrm{Fe}_{6}^{3+} M_{4 x}(\mathrm{OH})_{9}\left(\mathrm{H}_{2} \mathrm{O}\right)_{2}\left(\mathrm{PO}_{4}\right)_{5}$, where $M=\mathrm{Fe}^{3+}, \mathrm{Cu}^{2+}$ or other metal cation, and $x \approx$ 0.02 . This formula has 23.16 positive charges if only $\mathrm{Cu}^{2+}$ is present on the two $M$ sites, and 24 negative charges. If only $\mathrm{Fe}^{3+}$ would occupy the $M$ sites, a sum of 23.24 positive charges would result. However, the bond-valence sum of Oh(14), 0.87 v.u., could indicate that there might also be small amounts of water on this site and, consequently, $<24$ negative charges. Oh(14) does not accept any hydrogen bonds and can, therefore, not increase its bond-valence sum through hydrogen bonding. The non-stoichiometric nature of the formula is thus comparable to that in kidwellite.

The idealized formula of 'laubmannite' is $\left(\mathrm{Fe}^{3+}, \mathrm{Fe}^{2+}, M\right)_{8+x}\left(\mathrm{OH}, \mathrm{H}_{2} \mathrm{O}\right)_{9}\left(\mathrm{H}_{2} \mathrm{O}\right)_{2}\left(\mathrm{PO}_{4}\right)_{5}$, where $M=\mathrm{Fe}^{3+}, \mathrm{Cu}^{2+}$ or other metal cation, and $x \approx$ 0.1 . This formula has a distinctly lower Fe:P ratio (1.60) than the originally proposed formulae, $\mathrm{Fe}_{3}^{2+} \mathrm{Fe}_{6}^{3+}\left(\mathrm{PO}_{4}\right)_{4}(\mathrm{OH})_{12}$ (Frondel, 1949) and $\mathrm{Fe}_{9}\left(\mathrm{PO}_{4}\right)_{4}(\mathrm{OH})_{15}$ (Moore, 1970), and is intermediate between that of beraunite and kidwellite (both 1.50) and that of rockbridgeite and dufrénite (both 1.67). The similar ratios explain indirectly the often close association of 'laubmannite' with these other iron phosphates. The suggested hydrogen bonding scheme in 'laubmannite' (Table 9), which is based on the four detected $\mathrm{H}$ positions, shows that the hydrogen bonds are rather weak, with donor-acceptor distances all $>2.75(4) \AA$, similar to the situation in kidwellite (see above).

A powder diffraction pattern calculated from the structure model is in good agreement with that given by Moore (1970) (see also ICDD-PDF 22283). The calculated density, $3.37 \mathrm{~g} / \mathrm{cm}^{3}$ is relatively close to the value of $3.33 \mathrm{~g} / \mathrm{cm}^{3}$ measured for the laubmannite type material of Frondel (1949), which, however, represented a mixture of different iron phosphates (Moore, 1970; Dunn, 1990). 'Laubmannite' material from Buckeye Mountain, Arkansas, characterized by Moore (1970) gives a calculated density of $3.41 \mathrm{~g} / \mathrm{cm}^{3}$ on the basis of the formula $\mathrm{Fe}_{9}\left(\mathrm{PO}_{4}\right)_{4}(\mathrm{OH})_{15}$. The presently determined formula, $\left(\mathrm{Fe}^{3+}, \mathrm{Fe}^{2+}\right)_{2} \mathrm{Fe}_{6}^{3+}\left(\mathrm{PO}_{4}\right)_{5}(\mathrm{OH})_{8}\left(\mathrm{H}_{2} \mathrm{O}\right)_{3}$, results in a lower calculated density because it has a lower Fe:P ratio than that suggested by Moore (1970).

\section{Relation to other iron phosphates and explanation of epitaxial intergrowths}

The structure determinations of kidwellite and 'laubmannite' do not confirm the assumption of Moore (1970) that both minerals contain clusters composed of three face-sharing $\left(\mathrm{Fe}^{2+}, \mathrm{Fe}^{3+}\right) \mathrm{O}_{6}$ octahedra (' $h$-clusters'), which are present in the crystal structures of dufrénite and rockbridgeite (Moore, 1970). The conclusion of Moore (1970) was based on his observation that the occurrence of $\sim 13$ and $\sim 5.1 \AA$ axes are a common feature of several fibrous iron phosphates such as barbosalite, lipscombite, dufrénite, souzalite, rockbridgeite, beraunite, 'laubmannite' and kidwellite, and that his single-crystal study of 'laubmannite' had given the unit-cell parameters $a=13.95, b=30.77, c=$ $5.16 \AA$. Moore (1970) observed that the $\sim 13$ and $\sim 5.1 \AA$ axes always lie in the plane of dense polyhedral slabs.

The lengths of the $a$ axis in 'laubmannite', $5.17 \AA$, and the $b$ axis in kidwellite, $5.19 \AA$, are, in a rather fortuitous way, very similar to the those of the other iron phosphates mentioned. In rockbridgeite, the length of the $5.17 \AA$ axis is defined by the length of a face-sharing dimer, while in dufrénite the length of the $5.13 \AA$ axis 
represents the added lengths of two parallel polyhedral edges of an $\mathrm{FeO}_{6}$ octahedron and a $\mathrm{PO}_{4}$ tetrahedron (Moore, 1970). In both rockbridgeite and dufrénite the elongation of the ' $h$-cluster' is perpendicular to the respective $\sim 5.1 \AA$ axes.

Although 'laubmannite' does not contain ' $h$-clusters', the general connectivity in its structure also results in a thick polyhedral slab in the $a-b$ plane, i.e. in the plane of the 5.17 and $14.0 \AA$ axes. Adjacent slabs are connected solely via the edge-sharing dimer $\mathrm{Fe}(3) \mathrm{O}_{6}-\mathrm{Fe}(3) \mathrm{O}_{6}$. The cleavage observed on (010) may be explained by the fact that the weakest (i.e. corner-sharing) links are in a plane parallel to (010) (Fig. 3). In kidwellite, a similar, thick polyhedral slab is present in the $b-c$ plane, i.e. again in the plane of the 5.19 and $14.0 \AA$ axes. This slab includes the $\mathrm{NaO}_{6}$ polyhedron, and therefore differs from that in 'laubmannite'. The cleavage reported for kidwellite is parallel to the (100) plane (Moore and Ito, 1978). Thus, it may be explained by the comparatively weak bonds involving the $\mathrm{NaO}_{6}$ octahedron and the corner-sharing trimer (Fig. 1).

The frequently observed epitaxial intergrowth of kidwellite and 'laubmannite' and also intergrowths with other fibrous iron phosphates (see section 'Previous work') are explained by the overall similarity of the crystal structures.
Figure 5 gives a direct comparison of the similarly arranged, $\mathrm{FeO}_{6}$-based units in kidwellite and 'laubmannite' in views perpendicular to the $5.1 \AA$ axis, i.e. the morphological growth axis of both minerals (compare also Figs 2 and 4). It can be seen that the 'insertion' of the $\mathrm{Na}$ site in kidwellite induces, by comparison to 'laubmannite', a disruption of the edge-linkage of the two adjacent, face-sharing dimers. Simultaneously, an additional $\mathrm{FeO}_{6}$ octahedron is inserted between the two octahedra of the corner-sharing dimer to form the trimer in kidwellite. The resulting inserted layer is shown within a dashed box in Fig. 5. The similarity of the polyhedral building units and their arrangement makes it easy to understand that structural adaptations to changes in growth conditions (e.g. Fe:P ratio and $\mathrm{Na}^{+}$ activity in the solution, $\mathrm{pH}, \mathrm{Eh}$ ) of the fibrous iron phosphates discussed here can be accomplished by only small modifications of the polyhedral clusters (dimers, trimers, chains) and their interconnection (corner-, edge- or face-sharing).

\section{Acknowledgements}

The author thanks Mr Lothar Zapf of Zunsweier, Germany, Mr Richard Bayerl of Stuttgart, Germany, and Mr Carsten Slotta of Mühlenbach, Germany, for kindly providing the samples
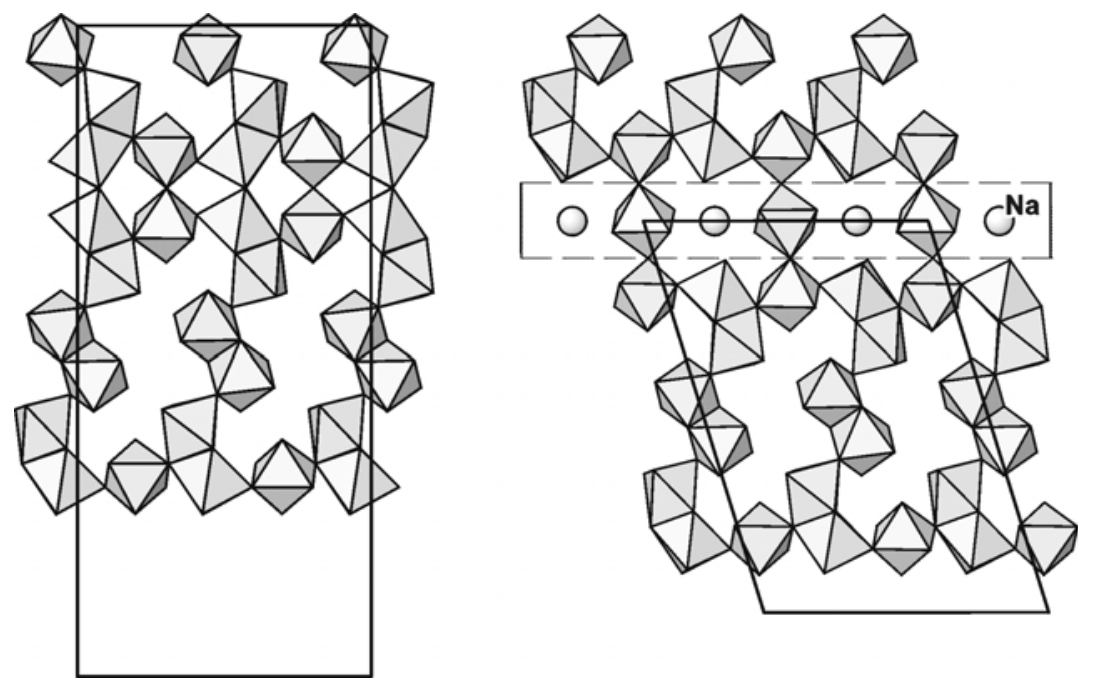

FIG. 5. Comparison of the $\mathrm{FeO}_{6}$-based building units in 'laubmannite' (left) and kidwellite (right) in views along the short $\sim 5.1 \AA$ axes, which represent the growth axes in both species. The similarity explains the often observed epitaxial intergrowths between both species and other related fibrous iron phosphates (see text for details and compare also with Figs 2 and 4). 
investigated here. Additional samples of 'laubmannite' were generously provided by Dennis Holmberg, Kiruna, Sweden, and Marco Ciriotti, Devesi-Cirié, Italy. Kia Wallwork and Allan Pring are thanked for providing some results of a Rietveld refinement of a kidwellite sample from Australia, which was based on the structure solution presented above. The article benefited from valuable comments by Mark Welch and an anonymous reviewer. The author gratefully acknowledges financial support of the Deutsche Forschungsgemeinschaft (DFG) via a Research Fellowship, and of the Austrian Science Foundation (FWF) (Grant P15220-GEO).

\section{References}

Anthony, J.W., Bideaux, R.A., Bladh, K.W. and Nichols, M.C. (2000) Handbook of Mineralogy. Vol. IV: Arsenates, Phosphates, Vanadates. Mineral Data Publishing, Tucson, Arizona, USA, 680 pp.

Barwood, H. (1974) Iron phosphate mineral locality at Indian Mountain, Alabama. Mineralogical Record, 5, 241-244.

Baur, W.H. (1981) Interatomic distance predictions for computer simulation of crystal structures. Pp. 31-52 in: Structure and Bonding in Crystals, Vol. II (M. O'Keeffe and A. Navrotsky, editors). Academic Press, New York.

Birch, W.D. (1990) Minerals from the Kintore and Block 14 opencuts, Broken Hill, N.S.W.; review of recent discoveries, including tsumebite, kipushite and otavite. Australian Mineralogist, 5, 125-141.

Birch, W.D., Pring, A., Self, P.G., Gibbs, R.B., Keck, E., Jensen, M.C. and Foord, E.E. (1996) Meurigite, a new fibrous iron phosphate resembling kidwellite. Mineralogical Magazine, 60, 787-793.

Bjällerud, C.G. (1989) Phosphate minerals from the Leveäniemi iron mine, Svappavaara, Sweden. Mineralogical Record, 20, 343-346.

Braithwaite, R.S.W. and Corke, H. (1980) Kidwellite from Cornwall. Mineralogical Magazine, 43, 952-953.

Brese, N.E. and O'Keeffe, M. (1991) Bond-valence parameters for solids. Acta Crystallographica, B 47, 192-197.

Dietrich, R. (1978a) Neues zur Phosphatparagenese der Grube Rotläufchen in Waldgirmes bei Wetzlar, Teil I. Aufschluss, 29, 107-124 (in German).

Dietrich, R. (1978b) Neues zur Phosphatparagenese der Grube Rotläufchen in Waldgirmes bei Wetzlar, Teil II. Aufschluss, 29, 139-153 (in German).

Dietrich, R. (1982) Die Mineralien der Phosphatparagenese der Grube Rotläufchen. Emser Hefte, 4(3), 22-47 (in German).
Dunn, P.J. (1990) Andrewsite and laubmannite formally discredited. American Mineralogist, 75, 1197-1199.

Frondel, C. (1949) The dufrenite problem. American Mineralogist, 34, 513-540.

Geipel, R. (1982) Sammeln in der Oberpfalz: Die Phosphatmineralien von Auerbach. MineralienMagazin, 6, 549-554 (in German).

Gibbs, R.B. (1991) New phosphate occurrences in southwestern New Mexico. New Mexico Geology, 13, 39-40.

Keller, P. (1985) Neue Mineralfunde aus dem Pegmatit von Sandamab, SWA/Namibia. Aufschluss, 36, 117-19 (in German).

Kolitsch, U. (1999) Evidence for the identity of meurigite and phosphofibrite by transmission electron microscopy and X-ray powder diffraction. European Journal of Mineralogy, 11, Beih. No. 1, 132.

Kolitsch, U. (2001) The atomic arrangement of the fibrous iron phosphate 'laubmannite' (as defined by Moore, 1970). European Journal of Mineralogy, 13, Beih. No. 1, 99.

Moore, P.B. (1970) Crystal chemistry of the basic iron phosphates. American Mineralogist, 55, 135-169.

Moore, P.B. and Ito, J. (1978) Kidwellite, $\mathrm{NaFe}^{3+}{ }_{9}(\mathrm{OH})_{10}\left(\mathrm{PO}_{4}\right)_{6} \cdot 5 \mathrm{H}_{2} \mathrm{O}$, a new species. Mineralogical Magazine, 42, 137-140.

Moore, P.B., Kampf, A.R. and Irving, A.J. (1974) Whitmoreite, $\mathrm{Fe}^{2+} \mathrm{Fe}^{3+}{ }_{2}(\mathrm{OH})_{2}\left(\mathrm{H}_{2} \mathrm{O}\right)_{4}\left[\mathrm{PO}_{4}\right]_{2}$, a new species: Its description and atomic arrangement. American Mineralogist, 59, 900-905.

Northrop, S.A. (1996) Minerals of New Mexico, $3^{\text {rd }}$ edition, revised by F.A. LaBruzza, University of New Mexico Press, Albuquerque, NM, USA, 346 pp.

Otwinowski, Z. and Minor, W. (1997) Processing of $\mathrm{X}$-ray diffraction data collected in oscillation mode. Methods in Enzymology, 276, 307-326.

Shape Software (1999) ATOMS for Windows and Macintosh V5.0.4. Kingsport, TN 37663, USA.

Sheldrick, G.M. (1997a) SHELXS-97, a Program for the Solution of Crystal Structures. University of Göttingen, Germany.

Sheldrick, G.M. (1997b) SHELXL-97, a program for crystal structure refinement. University of Göttingen, Germany.

Stanjek, H. (1983) Auerbach/Oberpfalz. Phosphatmineralien aus der Grube Leonie. Lapis, 8, 9-18; 42 (in German).

Vencato, I., Mascarenhas, Y.P. and Mattievich, E. (1986) The crystal structure of $\mathrm{Fe}^{2+} \mathrm{Fe}^{3+}{ }_{2}\left(\mathrm{PO}_{3} \mathrm{OH}\right)_{4}$ $\left(\mathrm{H}_{2} \mathrm{O}\right)_{4}$ : a new synthetic compound of mineralogic interest. American Mineralogist, 71, 222-226.

Vencato, I., Mattievich, E. and Mascarenhas, Y.P. (1989) Crystal structure of synthetic lipscombite: a redetermination. American Mineralogist, 74, $456-460$. 


\section{CRYSTAL STRUCTURES OF KIDWELLITE AND 'LAUBMANNITE'}

Walenta, K. (1981) Seltene Eisenphosphate aus der Grube Clara. Mineralien-Magazin, 5, 422-424 (in German).

Walenta, K. (1990) Neue Mineralfunde von der Grube Clara (4. Folge). Lapis, 15(4), 36-39.

Walenta, K. (1992) Die Mineralien des Schwarzwaldes. Chr. Weise Verlag, Munich, Germany, 336 pp. (in German).

Walenta, K. (1995) Neue Mineralfunde von der Grube Clara. 6. Folge, 1. Teil. Lapis, 20(5), 33-38 (in German).

Walenta, K. and Binder, W. (1980) Kidwellit und Dufrenit aus den Mines de Montmins bei Echassiéres (französisches Zentralmassiv). Aufschluss, 31, 51-54 (in German).

Walenta, K. and Dunn, P.J. (1984) Phosphofibrit, ein neues Eisenphosphat aus der Grube Clara im mittleren Schwarzwald (BRD). Chemie der Erde, 43, 11-16. (in German).

Witzke, T. and Giesler, T. (1997) Neufunde aus Sachsen (VI): Churchit-(Y), Cyrilovit, Faustit, Kidwellit, Meurigit und Nickelhexahydrit aus der Lausitz. Lapis, 22(9), 36-38; 58 (in German).

[Manuscript received 20 March 2003:

revised 15 November 2003] 